\title{
Avoided Phase Transitions and Glassy Dynamics In Geometrically Frustrated Systems and Non-Abelian Theories
}

\author{
Zohar Nussinov* \\ Institute Lorentz for Theoretical Physics, Leiden University \\ P.O.B. 9506, 2300 RA Leiden, The Netherlands \\ (October 24, 2018; E-mail:zohar@lorentz.leidenuniv.nl)
}

\begin{abstract}
We demonstrate that the application of any external uniform non-abelian gauge background, no matter how small, leads to a greatly enhanced degeneracy. This degeneracy is so large that even a non-abelian background field of infinitesimal strength leads to a shocking change in the thermodynamics. The critical temperature might be discontinuously depressed and an "avoided critical point" will emerge. We focus on how this arises in models previously employed to describe the microscopics of metallic glasses and correctly predicted the structure factor peaks. Some of the best fits, to date, to the dynamics of supercooled liquids were inspired by such notions for which we now provide a suggestive microscopic basis. We generalize the Mermin-Wagner inequality to high dimensions and discuss how extensive configurational entropy may be computed, by replica calculations, for a multitude of glass models (including non-Abelian gauge backgrounds). This extensive configurational entropy then allows a possible derivation of Vogel-Fulcher dynamics. We fortify earlier ideas suggesting avoided critical dynamics.
\end{abstract}

\section{INTRODUCTION}

Geometric frustration [1] spans a wide range of systems- Frank-Kasper phases [2] [3], [4], disordered materials, clathrates, and complex fluids such as the cholsteric blue phases [5], and amphiphilic membranes [6]. Perhaps most notable of these materials are metallic glasses, and possibly glasses in general, whose ideal icosahedral (and more complicated extensions) packing is not compatible with Euclidean space filling. Such packings have seen a revival in the study of quasicrystals. Till now many theoretical treatments, e.g. [7], have treated geometrical frustration as a perturbative effect akin to the smooth suppression of the superconductivity by the insertion of a frustrating magnetic field and the appearance of topological defects (vortices) in type II superconductors. A first hint that the physics might be far richer and reward us with interesting surprises was found in [10], [11] where the insertion of an infinite range Coulomb interaction was shown to be non-perturbative. By analogy to the $1 / \mathrm{r}$ gravitational potentials generated by geometrical deformations, all of this suggested that a similar occurrence might arise in geometrically frustrated systems.

Here we will show how an explicit infinitesimal geometric frustration may lead to a dramatic, non-perturbative, effect on the thermodynamics in a multitude of systems. As a result of geometric frustration, the critical temperature might be discontinuously depressed and an "avoided critical point" will emerge. The physical origin of the discontinuity is a greatly enhanced degeneracy: the geometric frustration thwarts ideal order (or crystallization) and leads to many competing low energy states. In this article we will focus on how this arises in various $O(n)$ systems immersed in uniform $S O(n)$ backgrounds. Similar models [5], [7] were previously employed to describe the microscopics of metallic glasses and correctly pre- dicted the locations of the structure factor peaks [7]. We will show that that such models exhibit "avoided critical behavior": a genuine true non-analyticity only at very low temperatures as compared to the (avoided) critical temperature of the system sans any frustrating external non-abelian gauge background. Some of the best fits [12], to date, to the dynamics of supercooled liquids were inspired by such notions for which we now provide a firmer microscopic basis. We will compare one of the fitted parameters in these plots (the magnitude of the "avoided critical temperature" for each of the supercooled liquids) and demonstrate that, on average, these might be well predicted by our microscopic picture.

To couch geometrical frustration in a general field theoretical setting, we will consider systems whose continuum Lagrangian density may be written in the form

$$
\mathcal{L}_{\text {matter }}=\frac{1}{2}\left|D_{\mu} \phi^{\nu}\right|^{2}-\frac{M^{2}}{2}|\vec{\phi}|^{2}+\frac{u}{4 !}|\vec{\phi}|^{4}+\ldots
$$

with a covariant derivative $D_{\mu}(x)=\partial_{\mu}-i \theta A_{\mu}(x)$ appropriate for parallel transport on a surface whose curvature is $(1 / R)=\theta$. As we will detail below, the strength of the coupling to the background gauge field $(\theta)$ sets the degree of geometric frustration present in the system.

Let us quickly sketch what unfolds as the frustration is introduced. Matters become most transparent in momentum space which diagonalizes the quadratic part of the action. When $\theta=0$, the quadratic part of the action has a single minimum at wavenumber $\vec{k}=0$. In this standard case, long wavelength (small $|\vec{k}|)$ fluctuations can destroy order in dimensions $d \leq 2$ and indeed they do so for continuous fields $\vec{\phi}$. In high dimensions $(d>2)$, order reasserts itself at temperatures $T \leq T_{c}=\mathcal{O}(1)$.

This picture undergoes dramatic ramifications as the frustrating field is switched on. As seen, in Fig.(1), the minimum in $\vec{k}$ space is no longer point like but rather 
extends over a (d-1) dimensional manifold whose radius is set by the geometrical frustration $\theta$. For any frustration $\theta$, no matter how small, the manifold of minimizing modes $M$ is a high dimensional object spanning many modes. All Fourier modes on this surface are degenerate leading to an exceptionally high configurational degeneracy in real space. In the aftermath, an effective one dimensional behavior results (which can be made precise in the $n \rightarrow \infty$ limit) with no ordering transition in sight. Only at the unfrustrated point $\theta=0$ does the minimizing manifold $M$ shrink to the origin, and our old intuition regarding the long wavelength fluctuations is regained.

For simplicity, we will mostly consider a translationally invariant $D_{\mu}$ with non-commuting uniform gauge connections $A_{\mu}$. Our results can be extended to systems with arbitrary non-uniform $D_{\mu}(x)$ in which case our analysis may be repeated with the Fourier basis index replaced by an index labeling the more complicated diagonalizing eigenbasis (the minimizing manifold $M$ will merely appear as an identical object in this basis).

We will consider non-Abelian gauge background fields $\left\{A_{\mu}(x)\right\}$ having no dynamics of their own and merely enforcing geometrical frustration. To make the gauge field a true dynamical variable, we will need to add terms involving derivatives; the simplest standard gauge invariant choice is the squared field $\left(F_{\mu \nu}\right)^{2}$ where the field $F_{\mu \nu}=\left[D_{\mu}, D_{\nu}\right]$. The resulting well known Lagrangian

$$
\begin{gathered}
\mathcal{L}_{\text {matter }}+\text { gauge } \\
=-\frac{1}{4} F_{\mu \nu} F^{\mu \nu} \\
+\frac{1}{2}\left|D_{\mu} \phi^{\nu}\right|^{2}-\frac{M^{2}}{2}|\vec{\phi}|^{2}+\frac{u}{4 !}|\vec{\phi}|^{4}+\ldots
\end{gathered}
$$

Theories involving only the first term are frequently referred to as "pure gauge theories". We will prove that in the opposite extreme of Eqn.(1) (non-dynamic or small $\left.A_{\mu}\right)$ a new surprising "avoided critical behavior" may occur: the effect of coupling to the non-Abelian gauges is non-perturbative. The theory suddenly becomes extremely sensitive to thermal effects. The density of states becomes, to a certain extent, one-dimensional. This also applies to some more general cases in which the effective action after integrating out the gauge fields will be of a form similar to Eqn.(1). For instance, if the kinetic term in the gauge fields is replaced by $\left(F_{\mu \nu}-f_{\mu \nu}\right)^{2}$ with specified frustrations $f_{\mu \nu}$ then after integrating out the gauge fields $A_{\mu}(x)$ the resulting action density in the matter fields $\vec{\phi}$ will exhibit frustration. Such a theoretical prediction has serious implications for many geometrically frustrated systems which will form a new universality class of their own. As we will aim to highlight in the paper, the very unusual "glass transition" might be linked at its very core to the non-perturbative physics spawned by non-Abelian background fields (although of a more complicated form than of a fixed uniform background).

Our main conclusion in the continuum limit is schematically summarized in the large $n$ phase diagram

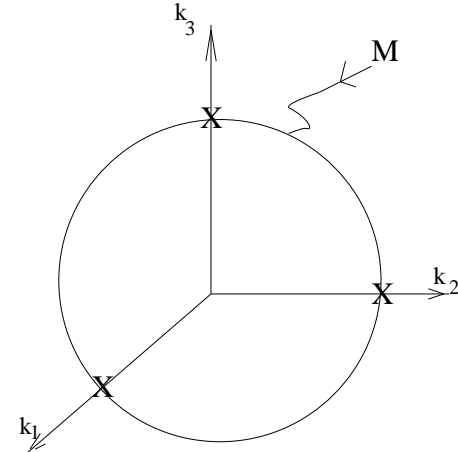

FIG. 1. A schematic of the interaction kernel, $v(\vec{k})$, minima in Fourier space. In all of the systems that we consider here, the relevant continuum limit $v(\vec{k})$ attains its minima on shells $|\vec{k}|=q>0$. In the presence of lattice point group symmetry terms, the rotational symmetry is lifted and the minima appear at a discrete number of points (labeled by " $\mathrm{X}$ " in the figure).

of Fig.(2). Here, the horizontal axis denotes the strength $\theta \equiv R^{-1}$ of a uniform non-abelian $S O(n)$ gauge background. The vertical axis denotes the critical temperature of a three dimensional $O(n)$ spin system. In the absence of a uniform non-Abelian background field (i.e. at $\theta=0)$, the spin system undergoes a phase transition at a finite $T=T_{c}(0)$ which is order of the exchange constant $J$. When a non-abelian background field is applied, the system is unable to order at finite temperatures.

We also report on a generalized Mermin-Wagner inequality which allows us to connect the magnitude of the order parameter (the absence of entropic effects) to the characteristic relaxation times present in any system (including glass models). We will also show how to derive Vogel-Fulcher dynamics for a multitude of glass models (including non-Abelian gauge backgrounds), and try to fortify earlier ideas suggesting avoided critical dynamics.

\section{DEFINITIONS AND CONVENTIONS}

Throughout, we will examine the interaction kernel $v$ appearing in the quadratic part of the Hamiltonian

$$
H=\frac{1}{2} \int \frac{d^{d} k}{(2 \pi)^{3}} v_{i j}(\vec{k}) \phi_{i}(\vec{k}) \phi_{j}(-\vec{k}) .
$$

This is the Fourier transform of the real space $H=$ $\frac{1}{2} \int d^{d} x \int d^{d} y V_{i j}(\vec{x}-\vec{y}) \phi_{i}(\vec{x}) \phi_{j}(\vec{y})$. For spins lattice systems we will consider Hamiltonians of the type

$$
H=\frac{1}{2} \sum_{\vec{x}, \vec{y}} V(\vec{x}-\vec{y})[\vec{S}(\vec{x}) \cdot \vec{S}(\vec{y})] .
$$

The sites $\vec{x}$ and $\vec{y}$ lie on a (generally hypercubic) lattice of size $N$, and $\left\{S_{i}(\vec{x})\right\}$ denote the $i$-th component of the spin $\vec{S}$ situated at a certain site $\vec{x}$. The normalized spins have $n$ components. Throughout, we employ 


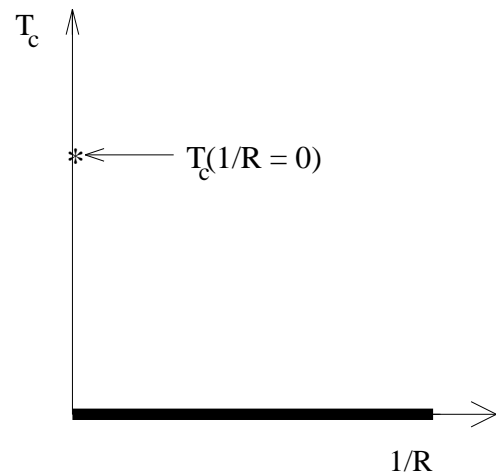

FIG. 2. The typical phase diagram encountered in $n \geq 2$ realizations of our models. When the system is unfrustrated, the critical temperature in dimensions $d>2$ is finite (and of order $\mathcal{O}(1))$. When the minimizing manifold $M$ is a $(d-1)$ dimensional shell, the critical temperature drops to zero. In the above, $1 / \mathrm{R}$ corresponds to the inverse radius of the sphere on which ideal order (icosahedral or other) can prevail. $1 / \mathrm{R}$ scales as $q$ - the radius of the minimizing shell $M$ is $\vec{k}$ space.

the non-symmetrical Fourier basis convention $(f(\vec{k})=$ $\left.\sum_{\vec{x}} F(\vec{x}) e^{-i \vec{k} \cdot \vec{x}} ; \quad F(\vec{x})=\frac{1}{N} \sum_{\vec{k}} f(\vec{k}) e^{i \vec{k} \cdot \vec{x}}\right)$. The vector $\vec{q}$ denotes a wave-vector minimizing the kernel $v(\vec{k})$ : $v(\vec{q})=\min _{k}\{v(\vec{k})\}$. In the usual ferromagnetic models $\vec{q}=0$ is the only minimizing mode. For simplicity, we set the lattice constant to unity and consider a lattice (of size $L)$ with periodic boundary conditions. The wave-vector components $k_{l}=\frac{2 \pi r_{l}}{L}$ where $r_{l}$ is an integer (and the real space coordinates $x_{l}$ are integers). In all systems that we study in this paper the Fourier modes $\vec{q}_{i}$ minimizing the interaction kernel $v(\vec{k})$ lie on a (d-1) dimensional shell. We often refer to this high dimensional minimizing manifold as $M$. The high dimensional $\vec{k}$ space redundancy leads to a complicated "energy landscape" for real space configurations. As there are many zero modes tangent to $M$ that cost no energy, the system is very fragile to perturbations and in the large $n$ limit, $T_{c}=0$. On a lattice, the continuous rotational symmetry is lifted. Here, we find a finite number of minimizing modes (denoted by an "X" in Fig.(1)). Notwithstanding, the critical temperature will typically be much lower than that of the unfrustrated system with a single minimum at $\vec{k}=0$. The critical temperature will drop discontinuously (from $T_{c}=O(1)$ to $\left.T_{c} \ll O(1)\right)$ as $M$ expands from the origin.

\section{ICOSAHEDRAL ORDER IN BRIEF}

Unlike other sections to follow, all presented here is a review of earlier work [1], [5], [7] which inspired the current publication. When a liquid is supercooled (when cooled so rapidly that it may not crystallize), and cooled further down until its relaxation times become effectively infinite it becomes a "glass". In slow solidification into an ordered crystal, the atoms/molecules readjust their posi- tions, slowly "computing" their ideal crystalline positions and veer toward them. Now, consider the situation, for the supercooled liquid, when the "computation" needs to be done almost on the spot. Here, the atoms/molecules can only probe their immediate surrounding to minimize energy costs of bonds with their nearest neighbors. For a Lennard-Jones interaction, each atom would like to be some distance $r_{\min }$ (the distance at which the Lennard Jones like potential $V(r)$ is minimized) away from all of its neighbors in order to minimize each bond individually. Here, crystallization is trivial in two dimensionsthe local minimum can be extended to tile the entire plane. Locally and globally, the ideal packing is hexagonal with each atom surrounded by its six nearest neighbors all equidistant from it at the ideal distance $r_{\min }$. The local minimum that the atoms may find is also the global minimum. Such a fortunate occurrence does not arise in three dimensions. Four atoms may be equidistant from each other to form the vertices of a perfect tetrahedron. However, around a given edge of the tetrahedron we may not pack five other tetrahedrons perfectly: We can almost do so leaving a $7^{\circ}$ void. Similarly, particles whose locally preferred structure is an icosahedron (of 13 particles) cannot globally tile all of space as seen by the "illegal" icosahedral five-fold rotational symmetry [16]. If this is correct, then microscopically, local clusters should appear. Certain supercooled metallic glasses offer a good testing ground for these notions. There is evidence that short-range order in under-cooled liquids and metallic glasses is icosahedral [8]. A theoretical construct invented long ago $[1,3,5]$ is a high dimensional reference system in which perfect local icosahedral order, with equal bond distances, could easily tile large regions without any voids. Endowing three dimensional space with a small curvature, the $7^{\circ}$ void can be compressed to zero: Icosahedral clustering is perfect in a slightly curved three dimensional space. An ideal, icosahedral crystal on the surface of a 4 -sphere (polytope " $\{3,3,5\}$ "), can be found. It consists of 120 particles embedded on the slightly curved spherical surface $S^{3}$ (the three dimensional boundary of a sphere in four dimensions). We ( [12]) have taken this notion to the extreme and argued that the dynamics of glasses is controlled by the vicinity to the melting transition of ideal crystal that may form on the curved sphere. Regions of short-range $\{3,3,5\}$ order in are broken up by an array of $-72^{\circ}$ disclination lines, forced in by "frustration" - the incompatibility of flat space with a space filling icosahedral crystal. The Frank-Kasper phases of transition metal alloys are ordered arrays of disclination lines in such an icosahedral medium. The order parameters $Q_{n, m_{a}, m_{b}}$ are obtained by projecting the local particle configuration onto the hyperspherical harmonics $Y_{n, m_{a}, m_{b}}$ (the spherical harmonics (" $Y_{l}^{m}$ "-s) for a 4-sphere) of a tangent four-sphere which can accommodate icosahedral order [7].

In three dimensions, a 3 -sphere is attached to every 


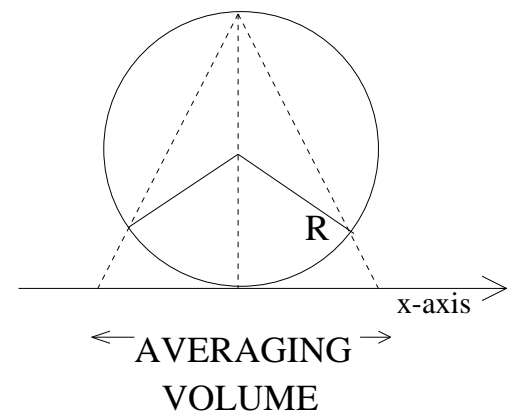

FIG. 3. A one dimensional analogue. We project the mass density from the real-space $x$-axis onto a disk of radius $R$ (with internal coordinate $\theta$ ) associated with each point on the $x$-axis and expand in harmonics $\left(\rho(x, \theta)=\sum_{m} \rho_{m}(x) e^{i m \theta}\right)$. Ideally, the mass density on the line matches that obtained by rolling the disk and the local energy functional $\left.\left[\partial_{x}-\partial_{\theta} / R\right] \rho(x, \theta)\right]^{2}$ is minimized. Integrating over $\theta$ we find the one dimensional analogue of the $S O(4)$ action below.

point $\vec{x}$ in real space. The mass density is projected onto these spheres. The density at all $\vec{x}$ on the tangent sphere ( $\hat{u}$ is the internal coordinate on the sphere),

$$
\rho(\vec{x}, \hat{u}) \equiv \sum_{n ; m_{+}, m_{-}} Q_{n ; m_{+}, m_{-}}^{*}(\vec{x}) Y_{n ; m_{+}, m_{-}}(\hat{u}),
$$

upon expansion in the hyper-spherical harmonics $Y_{n ; m_{+}, m_{-}}$. The $m_{ \pm}$subscripts in $Y_{n ; m_{+}, m_{-}}$refer to the two $S U(2)$ representations (of generators $\vec{A}_{ \pm}$of spin n/2) that the $S O(4)$ representation can be decomposed into. The Hamiltonian

$$
H=\frac{1}{2} \sum_{n}\left[K_{n}\left|\left(\partial_{\sigma}-\imath \theta L_{o \sigma}^{n}\right) \vec{Q}_{n}\right|^{2}+\mu_{n}|\vec{Q}|^{2}\right]
$$

where we organize the $\left\{Q_{n ; m_{+} m_{-}}\right\}$multipoles for each $n$ into $(n+1)^{2}$ dimensional vectors in the $\left|n / 2, m_{+} ; n / 2, m_{-}\right\rangle$basis. In a large $n$ analysis, the second (mass) term portrays the effect of normalization. Fourier transforming and diagonalizing [7],

$$
H=\frac{1}{2} \sum_{\vec{k}, M, i, n}\left|\alpha_{M, i}^{n}(\vec{k})\right|^{2}\left[K_{n} \lambda_{M, i}^{n}(\vec{k})+\mu_{n}\right],
$$

with $M$ the eigenvalues of $\hat{k} \cdot\left[\vec{A}_{+}+\vec{A}_{-}\right]$, the amplitudes of the i-th eigenmode $\alpha_{l}^{i}(\vec{k})$, and $\left\{\lambda_{M, i}^{n}(\vec{k})\right\}$ the eigenmode spectrum. Diagonalization yields, in all cases, a set of eigenvalues $\left\{\lambda_{n}^{i}(|\vec{k}|)\right\}$ with the global minimum (for each $n>0$ ) occurring at $k=q_{n}^{i}>0$, in agreement with structure factor measurements (displaying a series of peaks at these minimizing wave-vectors). By rotational symmetry, the eigenvalues depend on the magnitude $|\vec{k}|$ of the wavenumber. Thus, as the minima do not occur at the origin, shells of minimizing modes (as in Fig.(1)) occur. We now define

$$
\min _{n, i, \vec{k}}\left\{K_{n} \lambda_{n}^{i}(|\vec{k}|)\right\}=K_{n_{\min }} \lambda_{n_{\min }}^{i_{\min }}(q)
$$

where $n_{\min }$ and $i_{\min }$ denote the values of these indices at which the global minimum is attained- the corresponding minimizing wavenumber is denoted by $\vec{q}$. By rotational symmetry, all that matters is the modulus $q$. As expected, $q$ scales with the geometric frustration $\theta$. The values of $n$ allowed by icosahedral symmetry are $n=0,12,20,24,30,32,36$. The smallest non-trivial $n$ corresponds to a $(n+1)^{2}=169$ complex component vector $Q_{n, m_{+}, m_{-}}$. For a discussion on how these ideas may also be applied to wave functions on a sphere [14].

\section{NON-PERTURBATIVE THERMODYNAMICS}

We will argue, via a large $n$ analysis, a Mermin-Wagner inequality for similar models, and a fluctuation analysis [24], [33], that these and other continuum

$$
\text { glass models do not order at finite temperatures. }
$$

Any finite frustration $\theta$, no matter how small, drives these systems away from criticality at finite temperatures. In many solvable cases, this non-perturbative effect has nothing to do with the breakdown of perturbation theory due to long range interactions. For any value of the frustration $\theta$, the minimizing manifold of modes in Fourier space is high dimensional. The high dimensionality allows for the proliferation of many zero energy modes which easily erase any sign of potential order. When small point group symmetry terms lift the rotational symmetry of continuum models, the critical temperature becomes finite yet still minute as compared to the ordering temperature of the unfrustrated system,

$$
\mathcal{O}(1)=T_{c}(\theta=0) \gg T_{c}(\theta \neq 0) .
$$

\section{THE LARGE $N$ PHASE DIAGRAM}

Whenever the minimizing mode $q$ is finite and a (d1) shell of minimizing modes occurs (as in Fig.(1)), a large $n$ calculation reveals that the critical temperature, $T_{c}^{\text {Spherical }}=0$. As a function of the radius of the minimizing shell $M$ in Fig.(1), this drop occurs discontinuously.

In the large $n$ (or spherical) limit, we subject the system to a normalization constraint of the $(n+1)^{2}$ (that of $n=n_{\min }$ ) component vector $\vec{Q}$,

$$
\sum_{M, i, \vec{k}}\left|\alpha_{M, i}^{n_{\min }}(\vec{k})\right|^{2}=1
$$

We solve the system of Eqn.(7), subject to the latter (spherical or large $n$ ) constraint, to find that the critical temperature, 


$$
\frac{1}{k_{B} T_{c}}=\sum_{M, i} \int \frac{d^{3} k}{(2 \pi)^{3}} \frac{1}{K_{n_{\min }} \lambda_{M, i}^{n_{\min }}(\vec{k})-K_{n_{\min }} \lambda_{0, i_{\min }}^{n_{\min }}(q)} .
$$

As all terms in the integrand are explicitly positive, we may bound the right-hand side by its contribution from $\left(i_{\min }, M=0\right)$. If the $\left(i_{\min }, M=0\right)$ eigenvalue is analytic near its minimum at $|\vec{k}|=q$, then using the shorthand $F(|\vec{k}|) \equiv K_{n_{\min }} \lambda_{0, i_{\min }}^{n_{\min }}(\vec{k})$, a trivial bound (from the region $[q-\delta, q+\delta]$ ) reads

$$
\frac{1}{k_{B} T_{c}}>\frac{(q-\delta)^{2}}{\pi^{2}} \int_{q-\delta}^{q+\delta} d k \frac{1}{\left[F^{\prime \prime}(q)\right](k-q)^{2}}
$$

The integral in Eqn.(12) diverges. Here we assumed that the second derivative of $F$ is finite at $k=q$; if the second derivative vanishes then the divergence of the right-hand side of Eqn.(11) is even more dramatic. The divergence signals that the critical temperature $T_{c}=0$. Critics might argue that this might be an artifact of the large $n$ approximation and that, "for well known" reasons, this or that may happen (e.g. fluctuation driven first order phase transitions [35]). To counter such arguments, we prove in a later section a generalized Mermin-Wagner theorem of related rotationally symmetric spin models disallowing magnon dispersion relations about any ordered system at arbitrarily low temperatures $T=0^{+}$! Even if a fluctuation driven first order transition occurs, as we show (by extending replica calculations), the system may be frozen in a glass state before reaching the transition, so its existence might be immaterial. For the moment, we simply note that the lowest lying vector $\vec{Q}_{n=12}$ has 169 complex components. This number is large and a $1 / n$ expansion seems plausible. In contrast to certain expectations, it is seen that many beautiful early constructs $[1,7,5]$ predict a vanishing $T_{c}$ for any finite non-Abelian coupling if no symmetry breaking terms are present. As shown in Fig.(2), the phase diagram corresponding to these ideal metallic glasses, and plausibly to dense random packed supercooled liquids in general, shows an "avoided critical point" - a spike for tunable $\vec{q}=0$ in the interaction kernel, a genuine non-analyticity in the (thermo)dynamic functions at finite coupling to the uniform gauge background only at $T_{c}=0$.

\section{AVOIDED CRITICALITY VIA 1/N}

Within a $1 / n$ diagrammatic framework, the absence of a finite temperature phase transition, in the large $n$ limit, is seen by the divergence of the lowest order (tadpole) contribution to the self energy (Fig.4). With the selfconsistent propagators,

$$
G_{i_{\min }, n_{\min }}^{-1}(\vec{k})=K_{n_{\min }} \lambda_{0, i_{\min }}^{n_{\min }}(\vec{k})+r
$$

for the lowest energy mode $\left(n_{\min }, i_{\text {min }}\right)$ of relevance at low temperatures, with the bare propagator

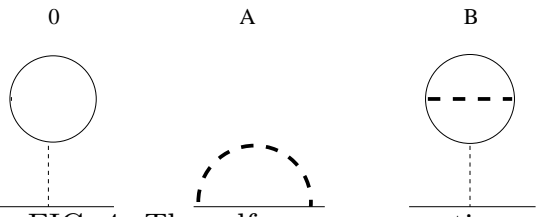

FIG. 4. The self energy corrections. The thin dashed lines denote bare interactions. Thick dashed lines represent dressed interactions (i.e. the bare interactions screened by a geometric series of bubble diagrams). The solid lines denote propagators. $\Sigma^{0}$ is the $O(1)$ parquet diagram. $\Sigma^{(A),(B)}$ are $O(1 / n)$ corrections.

$$
G_{0}^{-1}(\vec{k})=K_{n_{\min }} \lambda_{0, i_{\min }}^{n_{\min }}(\vec{k})+r_{0}
$$

The zeroth order self-energy is a momentum independent constant

$$
\Sigma^{(0)}=\int \frac{d^{3} k}{(2 \pi)^{3}} G_{i_{\min }, n_{\min }}(\vec{k}) .
$$

The zeroth order self-energy contributions from all other (non- $\left.\left(n_{m i n}, i_{m i n}\right)\right)$ eigenvalues are strictly positive. When the mass gap vanishes, i.e. $r_{\min }=$ $-K_{n_{\min }} \lambda_{0, i_{\text {min }}}^{n_{\min }}(q)$, the integral in Eqn.(15) diverges. By the Dyson equation,

$$
G^{-1}(\vec{k})=G_{0}^{-1}(\vec{k})+\Sigma(\vec{k})
$$

A divergent $\Sigma^{(0)} \sim\left(r-r_{\text {min }}\right)^{-1 / 2}$ signals the inability of the mass gap to vanish. Only at $r_{0}=-\infty$ can $r=-K_{n_{\min }} \lambda_{0, i_{\min }}^{n_{\min }}(q)$. In the conventional (finite $T_{c}$ ) case, the bare mass $r_{0}=a\left(T-T_{0}\right)$ codes for a linear temperature scale. The divergence that we obtain here implies that $T_{c}=0$ and that consequently a linear temperature scale is void in its environs. A similar analysis for the quantum case is detailed in the appendix. There are two first order $1 / \mathrm{n}$ diagrams and, after regrouping diagrams self-consistently, six important second order diagrams). By re-summing [24] diagrams self-consistently, the zeroth order divergence forbidding finite temperature ordering may stay in tact.

\section{NEW $S O(N)$ BACKGROUND SPIN MODELS}

Here we, by inventing a simple "soccer-ball model", we intuitively illustrate a simple basic premise:

A non-Abelian background leads to High Dimensional minimizing shells in $\vec{k}$-space.

The radius of the shell is set by the strength of the non-Abelian background. The profound increase in the dimensionality of the minimizing modes leads to a highly non-perturbative effect on the thermodynamics. In momentum space, there are many zero-energy, "Goldstone" like, modes tangent to the minimizing manifold. These 
fluctuations lead to a vanishing spin stiffness. All of this is in stark contrast to the innocuous effect of abelian gauge backgrounds: A $U(1)$ minimal substitution $v(\vec{k})=$ $(\vec{k}-e \vec{A})^{2}$, with a constant $\vec{A}$, trivially shifts the point minimum in $\vec{k}$ - space. For non-Abelian coupling the effect is much more subtle: the minimizing manifold is a shell spanning an infinite number of Fourier points. As the original models of [7] only allow for representations of $\mathrm{SO}(3)$ or $\mathrm{SO}(4)$ with a high number of components (large $\mathrm{n}$ indeed) for which calculational results may not be too transparent, we will now invent a simple toy spin model to gain intuition: the "soccer-ball model". The interplay between the external spatial and internal spin direction that it possesses is present in very few other spin systems of a very different character (e.g. orbital Jahn-Teller and Kugel-Khomskii like compass models). Consider three component (Heisenberg) spins on the square lattice with $\mathrm{SO}(3)$ couplings:

$$
H=-\sum_{\langle x, y\rangle} \vec{S}(\vec{x}) * \vec{S}(\vec{y}) .
$$

Here the $*$ operation denotes to the scalar product between a spin $\vec{S}(\vec{x})$ attached to the south pole a sphere (a "soccer-ball") and subsequently rolled to the neighboring site $\vec{y}$ and the spin $\vec{S}(\vec{y})$ situated at $\vec{y}$; the action is locally minimized by a spin rotation in the $[\ell n]$ plane for a lattice displacement along the $\ell$-axis. For a rolling angle $\theta$, we will now consider a spin configuration having as its only non-vanishing Fourier components $\vec{S}\left( \pm \theta \hat{e_{1}}\right)$, namely, the configuration

$$
\left(S_{1}, S_{2}\right)=\left(\cos \theta x_{1}, \sin \theta x_{1}\right)
$$

with $S_{1}$ and $S_{2}$ the x and y components of the spin and $x_{1}$ the spatial $\mathrm{x}$ coordinate. This spiral configuration will saturate all bonds along $\hat{e}_{1}$, and satisfy $\vec{S}(\vec{x})=\vec{S}\left(\vec{x}+\hat{e}_{2}\right)$ (just as for the uniform $(\vec{k}=0)$ configuration). The energy of the above configuration is lower than that of a $\vec{k}=0$ mode. In the continuum limit, the kernel is invariant under rotations of $\vec{k}$. As the minimum does not occur at the origin, a shell of minimizing modes is seen to exist (a $(d-w)$ dimensional manifold of minimizing modes is generated for $O(n=d+2-w)$ spins with the above "rolling" $S O(n)$ action for all but $(w-1)$ directions assigned ferromagnetic couplings). Alternatively, in the continuum limit, the differential action evaluated for the above configuration along any ray not parallel to $\hat{e}_{2}$ is lower than that for the uniform configuration. The continuum Hamiltonian

$$
H=\frac{1}{2} \int\left|D_{\mu} S^{\nu}\right|^{2} d^{2} x=\frac{1}{2} \int S_{i}(\vec{k}) v_{i, j}(\vec{k}) S_{j}(-\vec{k}) d^{2} k,
$$

a nonlinear $\sigma$ model on a curved surface. The covariant derivative

$$
D_{\mu}=\partial_{\mu}+\imath \theta \epsilon_{\mu \nu} \hat{L}_{\nu}
$$

where $L_{\nu}$ is the $\nu$ component of the $\ell=1$ angular momentum generator $\left(\epsilon_{12}=-\epsilon_{21}=1\right)$. Demanding that the "rolled" vector increment be real suggests the angular momentum representation $\left(\hat{L}_{i}\right)_{j k}=-\imath \epsilon_{i j k}$. The interaction kernel pursues the form:

$$
v_{i, j}(\vec{k})=k^{2} \delta_{i, j}+2 \theta \epsilon_{\mu \nu} k_{\mu}\left(L_{\nu}\right)_{i, j}+\theta^{2}\left(L_{x}^{2}+L_{y}^{2}\right)_{i, j} .
$$

The eigenvalues are invariant under planar rotations of $\vec{k}$. The minima occur on the ((2-1)dimensional) shell:

$$
\vec{q} \in M_{\theta}: \vec{q}^{2}=A \theta^{2} .
$$

For the lattice version $v_{i j}(\vec{k})=\sum_{ \pm \ell} e^{ \pm \imath k_{\ell}} R_{\ell}( \pm \theta)$ where $R_{\ell}( \pm \theta)$ is the rolling matrix (rotation matrix about the $\ell^{\natural} \neq \ell$-axis ). The continuum representation $L_{i}=-i \epsilon_{i j k}$ (for the change of the spin $\delta \vec{S}=\delta \vec{\phi} \times \vec{S}$ incurred by a rotation of angle $\delta \vec{\phi}$ ) emerges trivially.

Exploiting rotational invariance in the continuum limit, we may always rotate a pure mode $\vec{q}$ of unit $(O(n))$ spins such that it lies parallel to $\hat{e}_{1}$. All bonds parallel to $\hat{e}_{2}$ will entail the same energy penalty as the $\vec{k}=0$ mode and to minimize energy cost along $\hat{e}_{1}$ we simply set $\vec{q}=\theta \hat{e}_{1}$. We now relax the constraints of locally normalized real spins and allow the spins to be complex and only satisfy a global normalization constraint. Any ground states found here will obviously be of equal or lower energy than those of three-component Heisenberg spins. In a spherical model of complex spins with global normalization: $\sum_{\vec{x}}|\vec{S}(\vec{x})|^{2}=N$ enforced by a Lagrange multiplier $\mu$, the continuum limit kernel reads

$$
\left(\begin{array}{ccc}
k^{2}+\theta^{2}+\mu & 0 & 2 \imath \theta k_{y} \\
0 & k^{2}+\theta^{2}+\mu & 2 \imath \theta k_{x} \\
-2 \imath \theta k_{y} & -2 \imath \theta k_{x} & k^{2}+2 \theta^{2}+\mu
\end{array}\right) .
$$

Due to an inherent coupling between real and imaginary components, $|\vec{q}|=\theta$ may not be enforced.

As they must, all eigenvalues degenerate onto the canonical $k^{2}$ dispersion in the limit $\theta \rightarrow 0$. Two of the eigenvalues

$$
\begin{array}{r}
\lambda_{o}^{\prime} \equiv \lambda_{o}+\mu=k^{2}+\theta^{2}+\mu, \\
\lambda_{ \pm}^{\prime} \equiv \lambda_{ \pm}+\mu=\mu+k^{2}+\frac{3}{2} \theta^{2} \pm \frac{1}{2} \sqrt{16 k^{2} \theta^{2}+\theta^{4}},
\end{array}
$$

are non-analytic in $|\vec{k}|$. The global minimum

$$
\min _{\vec{k}}\left\{\lambda_{-}^{\prime}\right\}=\frac{7}{16} \theta^{2}+\mu
$$

occurs on the (2-1) dimensional annulus $|\vec{q}|=\frac{\sqrt{15}}{4}|\theta|$. The corresponding eigenvector is

$$
\begin{array}{r}
\left|u_{-}\right\rangle=\mathcal{N} \mid 1, \frac{-2 i \theta k_{x}}{-\theta^{2} / 2+\frac{1}{2} \theta \sqrt{16 k^{2}+\theta^{2}}}, \\
\left.\frac{-2 i \theta k_{y}}{-\theta^{2} / 2+\frac{1}{2} \theta \sqrt{16 k^{2}+\theta^{2}}}\right\rangle \\
\equiv\left|s_{1 ;-}^{R}, s_{2 ;-}^{I}, s_{3 ;-}^{I}\right\rangle
\end{array}
$$


where the $R, I$ superscripts denote real/imaginary components respectively. The minimum of the nonoscillatory "ferromagnetic" mode occurs at the origin ,

$$
\min _{\vec{k}}\left\{\lambda_{o}^{\prime}\right\}=\lambda_{o}^{\prime}(k=0)=\mu+\theta^{2} .
$$

A Landau-Ginzburg type analysis ( [24], [33]) shows strong (non-logarithmic) divergent fluctuations from $\vec{k} \sim$ $\vec{q}$ in directions (within the 6-dimensional parameter space $\left.\left(s_{1}^{R, I}, s_{2}^{R, I}, s_{3}^{R, I}\right)\right)$ transverse to the eigenvector of Eqn.(25) (such that the norm $|\vec{s}|$ does not change to lowest order). The generalization of our "soccer-ball" model to $\mathrm{SO}(4)$ couplings on a three dimensional lattice is discussed in Appendix(B). The reader might be dismayed that here we analyzed a situation in which the spins are complex. Here we attempt to capture as best as possible the complicated non-Abelian theories of glasses. In the actual high dimensional multipole expansion as in Eqs.(6) the "spin components" $\vec{Q}$ are complex.

\section{OTHER INTERACTIONS}

Non-Abelian background fields are not the only potential sources of glass formation and amorphous "clump" configurations. Many more physical Euclidean space potentials seem to do the trick. All of these interactions seem to share the generic feature that in Fourier space- the minimizing modes form a high dimensional shell (Fig.(1)) leading to an extremely high degeneracy in real space. This in turn leads to a "complex energy landscape" with many competing minima.

\section{A. The Coulomb Frustrated Ferromagnet}

My collaborators and I have [12] modeled the geometric frustration felt by molecules as they attempt to pack into a locally preferred structure by a long-range coulomb interaction forbidding the ideal local structure to tile all of space. In the continuum limit, the Hamiltonian

$$
\begin{aligned}
& H=\int d^{3} x\left[\frac{1}{2}(\nabla \phi)^{2}+\frac{m_{0}^{2}}{2} \phi^{2}+\frac{u}{4 !} \phi^{4}\right] \\
& +\int d^{3} x \int d^{3} x^{\prime} \phi(x) V\left(x-x^{\prime}\right) \phi\left(x^{\prime}\right),
\end{aligned}
$$

with the potential $V(r)=e^{2} / r$. The minimizing modes occur on shells as in Fig.(1) with $|\vec{q}| \simeq e^{1 / 2}$. Here we will derive such a model from the underlying microscopics.

\section{B. Kac-type Potentials}

These two-body interactions,

$$
V(|\vec{x}|)=V_{0} \gamma^{d} \phi(\gamma|\vec{x}|)
$$

with the step function $\phi(y)=1$ for $y \leq 1$ and zero otherwise and $V_{0}=\alpha^{2}$ [22]. The constant $\alpha$ controls the integral strength of the potential and $\gamma$ dictates the range of the potential. We note that, physically, this potential corresponds to a weak core interaction inside a sphere of radius $\gamma^{-1}$. These interactions may model glass formation [19]. The Fourier transformed interaction is minimized at finite wave-numbers (as easily seen, it has a maximum at $k=0$ when all the Fourier phases add coherently). The Fourier transform of Eqn.(28),

$$
v(|\vec{k}|)=4 \pi V_{0} \gamma^{3}[\sin (k / \gamma)-(k / \gamma) \cos (k / \gamma)] / k^{3} .
$$

This kernel displays its minimum at $k=q>0$ with $q / \gamma \simeq 5$.76. The minimum indeed occurs on a $(d-1)$ dimensional shell such as that drawn in Fig.(1). The kernel $v(k)$ is analytic near $|\vec{k}|=q$ allowing us to Taylor expand about the minimum. By superposing Kac-type potentials, we may generate discrete, "digitized", versions of the Lennard-Jones potential. For instance,

$$
V(\vec{x})=\sum_{i} V_{i} \gamma_{i}^{d} \phi\left(\gamma_{i}|\vec{x}|\right),
$$

with $V_{0}=\alpha^{2} \gg-V_{1}>0, \gamma_{1}=1 / r_{1}$, and $\gamma_{2}=1 / r_{2}$ corresponds to a potential $V(\vec{x})$ which is $\left(V_{0}+V_{1}\right) \gg 0$ inside a sphere of radius $r_{1}$ and is $V_{1}<0$ for $r_{1}<r \leq r_{2}$, and vanishes for all $r>r_{2}$. Superposing the Fourier transformed kernels of Eqn.(29), shows a minimum on a spherical shell of finite radius $|\vec{k}|=q>0$. Although the absolute minimum of a particles interacting via a Lennard-Jones is an FCC crystal, whenever the liquid is cooled rapidly it effectively sees the radially symmetric finite radius Fourier modes as the minimizing modes.

\section{Z1 and Z2 potentials}

Lately, two new potentials for monatomic glass like formers in the bulk were found [20]. In their presence, the atoms display non-compact arrangements of 13-atom icosahedra and a hint of fragile glassy dynamics in simulations of the cooled liquid state. These are [20]

$$
V(|\vec{r}|)=a \exp [-\alpha r] / r^{3} \cos \left(2 k_{F} r\right)+b(\sigma / r)^{n}+V_{0},
$$

with certain numerical values for the various parameters $\left\{a, \alpha, k_{F}, \sigma, n, V_{0}\right\}$ for $r>r_{c}$. This model can be related to a superposition of Kac-type potentials. As in all glass models that we investigate, the minimizing Fourier modes of $v(\vec{k})$ occur on shells. Like other potentials, $V(r)$ naturally leads to icosahedral like ordering over large non-compact regions. In the continuum limit, these potentials are akin to non-Abelian backgrounds. The extended space that they occupy offers a genuine 


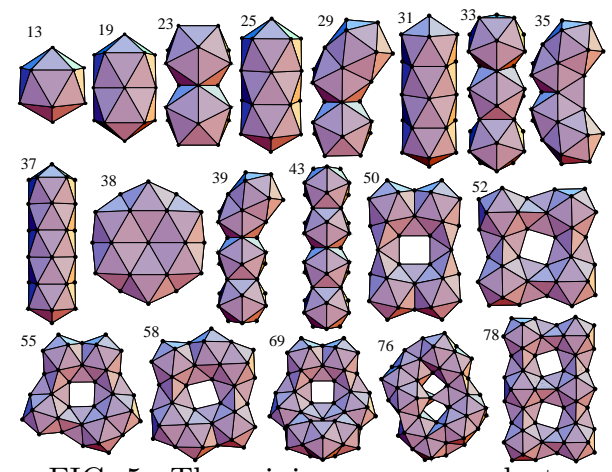

FIG. 5. The minimum energy clusters of the Z1 minima. The number next to each cluster denotes the number of atoms in it. This figure is reproduced from Doye et al.

example (not merely that of small 13 particle clusters) of relatively large systems $\left(\sim 10^{2}\right)$ with geometric frustration. The local maxima of the potential inhibit closed packed structures and lead to polytetrahedral structures which may be divided into tetrahedra with atoms at their corners. Local icosahedral order involves no disclinations but as the clusters enlarge, negative disclinations must be introduced. The disclination lines cannot end abruptly and must form closed loops or traverse the cluster. As in Frank-Kasper phases, the packing can be described by a network of disclination lines threading an icosahedrally coordinated medium. Clusters of various sizes are depicted in Fig.(5) from [20]. For the two potentials for the largest clusters, networks and chains of icosahedra are respectively preferred. Upon cooling, in silico, a liquid of $\sim 10^{4}$ particles, extended icosahedral clusters form with long time for local rearrangements. These potentials provide a natural realization of the notions underlying geometric frustration (here with icosahedral like order over a large range), and a naturally related glassy dynamics (slow rearrangement of icosahedral aggregates inhibiting crystallization leading to better glass-formers).

\section{CORRELATORS AND DISORDER LINES}

\section{A. The $n \rightarrow \infty$ limit}

The correlation functions (and much of the physics) are governed by the "thermal" dynamics of the zeros and branch cuts of the energy spectrum in the complex $\mathrm{k}$ plane. Generically, in a system with many competing interactions- with either direct competition (as in the Coulomb frustrated ferromagnet) or indirect (e.g. nonAbelian backgrounds)- the Green functions will display numerous poles and/or branch points. These singularities, generally, adiabatically shift their location in the complex plane as a function of temperature (the chemical potential $\mu$ or mass gap $r$ ). Exactly how they evolve in the complex $k$ plane determines, to a large extent, the nature of the transitions and crossovers that occur.

In all of the models that we consider- all models with a minimum of the kernel on a shell in $|k|$ space- we find that at low temperatures the branch point or pole of relevance has a finite real part leading to oscillations. This is expected as at low temperatures, the relevant Fourier modes are on the shell $|\vec{k}|=q$ and the system must display oscillations. As temperature is increased, the modulation length becomes longer and longer. The evolution of the modulation length can be either continuous or discontinuous. For systems with finite range interactions (including non-Abelian backgrounds), the number of poles and branch-points is conserved as a function of temperature- no two (or more) singularities merge in the complex plane. In such systems, the modulation length may, at times, jump from one value to another. This occurs when one pole or branch point approaches the real axis more than another singularity (and consequently has a smaller imaginary part). The relevant asymptotic modulation length then jumps to the singularity which is closer to the real axis. In our toy $\mathrm{SO}(3)$ actions, this indeed happens: At high temperatures, the system is, asymptotically unmodulated. Below a crossover temperature, the system jumps and starts to exhibit finite modulations associated with singularities of another eigenvalue. Naively, one might expect the branch points present in the eigenvalue spectrum to immediately lead to algebraic (i.e. non-exponential) decay of correlations at all temperatures. This, however, is not what occurs. Cancelations arise and correlations decay exponentially at all finite temperatures (as expected).

In systems with competing interactions of different longer range interactions, the poles merge frequently. For instance, in the Coulomb Frustrated Ferromagnet, the poles move along the imaginary axis at high temperatures. At a certain crossover temperature, they merge and hit the "unit" circle. From there, the poles bifurcate in pairs to form two conjugate pairs of poles on the unit circle leading to finite modulations which become of shorter and shorter length (and ever larger correlation length) as the temperature is decreased. The merger of the poles at the crossover temperature leads to a "disorder line" like effect. The thermodynamic functions are analytic at this temperature yet their explicit real functional form changes (just an analytic continuation from one natural set of functions to another). In systems with long range interactions (e.g. the Coulomb Frustrated Ferromagnet of Eqn.(27))), the modulation lengths tend to decrease as temperature is decreased; the converse occurs for the canonical interactions $\left(k^{2}\right)$ thwarted or frustrated by an even shorter range interactions (e.g. $k^{4}$ ). In physical terms, the crossover (disorder line) temperature is related to a temperature that marks the gradual crossover from a one dimensional system (the relevant density of states at low temperatures is indeed one dimensional) to a canonical three dimensional system at 
high temperatures. For explicit expressions for the correlation and modulation length crossovers the reader us referred to Appendix(C). A discussion of the thermodynamic crossovers appears in $\operatorname{Appendix}(D)$.

\section{B. A Josephson like length}

At very low temperatures the real space correlation function $G(\vec{x})$ decays differently in different regimes. A $O(1 / n)$ calculation reveals an algebraic cross-over similar to an inverted Josephson like regime. Specifically, the pair correlator

$$
\begin{array}{r}
G(\vec{x}) \sim|\vec{x}|^{-2} \text { for } \ell_{J} \ll|\vec{x}| \ll L_{D} \ll \xi, \\
G(\vec{x}) \sim|\vec{x}|^{-1} \text { for }|\vec{x}| \ll \ell_{J},
\end{array}
$$

with the Josephson like length

$$
\ell_{J} \equiv n /\left(2 \Sigma^{(0)}\right)
$$

Evaluating matters explicitly ( [11], [24]), we find that the Josephson like correlation length $\ell_{J}$ scales as the inverse of the correlation length in the frustrated system. Here $\Sigma^{(0)}$ is the $\mathcal{O}\left(n^{0}\right)$ self-energy, $\xi$ is the correlation length, and $L_{D}$, the domain size, is $\left(2 \pi /\left|\operatorname{Re}\left\{k_{-}\right\}\right|\right)$, with "Re" denoting the real part. More generally. within the general $\mathrm{SO}(4)$ theory, $k_{-}$is replaced by the root of the eigenvalue corresponding the largest correlation length (smallest imaginary part). This length scale appears for all actions in which the relevant interaction kernel is peaked about a (d-1) dimensional manifold $|\vec{k}|=q>0$ about which they are quadratic.

\section{A GENERALIZED MERMIN-WAGNER INEQUALITY}

Here we report on an extension of the Mermin-Wagner theorem which naturally links the magnitude of the order parameter to constraints on the characteristic decoherence or relaxation time. We generalized the MerminWagner inequality to all two dimensional systems with an analytic interaction kernel and to a host of three $d i$ mensional systems in which a certain integral diverges. The inequality that we derive reads

$$
2\left|m_{q}\right|^{2} T\left\langle\tau_{\vec{k}}\right\rangle \leq 1
$$

with $m_{q}$ the magnetization at the minimizing momentum $q$, and (with $\hbar=1$ ) $\tau_{\vec{k}}$ is an integral having the dimensions of time reflecting the characteristic decoherence or relaxation time in a quantum system suffering a boost of momentum $\vec{k}$ (scaled magnon liftime). If the characteristic relaxation time diverges the system does not admit, at any finite temperature, a fluctuation spectrum about an ordered state. As we will later show becomes glassy (its configurational entropy diverges). The characteristic decoherence time

$$
1 /\left(\Delta_{\vec{k}}^{(2)} E\right) \equiv \tau_{\vec{k}}
$$

with $\Delta_{\vec{k}}^{(2)} E$ the change of energy of the system due to a boost of momentum $\vec{k}$ or scaled spin-wave energy. Whenever the integral

$$
\frac{1}{(2 \pi)^{d}} \int \frac{d^{d} k}{v(\vec{k}+\vec{q})+v(\vec{k}-\vec{q})-2 v(\vec{q})}
$$

diverges then we may not obtain the low temperature $T=0^{+}$magnon dispersion by assuming a nearly perfectly order state. In the above, $\vec{q} \in M$ is any vector on the minimizing manifold $(M)$ in momentum space. For further details the reader is referred to the Appendices. In Appendix(E) we generalize the Mermin-Wagner inequality to all rotationally invariant two dimensional system with an analytic interaction kernel in momentum space. In $\operatorname{Appendix}(\mathrm{F})$, we derive inequalities in three dimensions and interpret the extended MerminWagner theorem in terms of relaxation times. When multiplied by the temperature, the integral appearing in the generalized Mermin-Wagner inequality (Eqn.(35)) is a strict lower bound on thermal fluctuations. Spin systems with a non-trivial minimizing manifold in $\vec{k}$ space, much like spin ladders, display an interesting even-odd effect. When we analyze the fluctuations of spins having an even number of components (n), we find precisely the integral of Eqn.(35) appearing as the relevant thermal fluctuation integral. For a system of spins having an odd number of components, we find a more divergent (for onshell minima in Fourier space) fluctuation integral. For further details the reader is referred to [33]. The bound that we derive by a generalized Mermin-Wagner inequality is a strict lower bound on the fluctuations. When the number of spin components $(n)$ is odd, the thermal fluctuations are much larger, and ordering is even more inhibited.

\section{ENTROPY AND DEGENERACIES}

The generalized Mermin-Wagner inequality suggests, divergent decoherence times are tied to a vanishing order parameter. Physically both effects stem from one originthe complicated energy landscape that these systems possess. The high degeneracy and near degeneracy of low lying states makes these systems vulnerable to thermal fluctuations. Metastability is at the heart of the matter. It is not surprising that the configurational entropy may be extensive at high enough temperatures. 


\section{A. Ground State Degeneracies}

The ground state degeneracies of this system are obvious. As the energy dispersion in these systems is effectively one dimensional (it depends only on the radial coordinate $|\vec{k}|)$, whereas the system is $d$ dimensional, the ground state degeneracy should be exponential in $L^{d-1}$, with $L$ the system size. These statements are exact in the spherical (large $n$ ) limit where every normalized superposition of minimizing modes constitutes a ground state. As the number of minimizing modes is $\mathcal{O}\left(L^{d-1}\right)$ [33], the previous exponential degeneracy follows. For special Ising models, we can rigorously prove [33] that the degeneracy is bounded from below by $2^{|M|}$ where $|M|$ denotes the number of points on the minimizing manifold which is characteristically $\mathcal{O}\left(L^{d-1}\right)$. For a similar analysis for $O(n)$ ground states with a trivial bound $L^{n(d-1)}$ see [33]. In the large $n$ limit all of this becomes exact and the entropy scales as the surface area leading to a holographic like ground state degeneracy.

\section{B. Replica Calculations}

For metastable states at higher temperatures the problem is harder and we were not able to derive rigorous results. A replica calculation within the self-consistent screening approximation reveals that the configurational entropy in all of these systems: continuum non-abelian gauge backgrounds, Kac-type interactions [22], Coulomb frustrations [21], and in general any system in which the interactions are peaked on (d-1) dimensional shell in Fourier space, is extensive.

The case of Coulomb frustration was investigated by [21] and generalized to Kac-type step-function interactions in [22]. Here we note that the minimal ingredient in all of these calculations, the requirement that the unperturbed Green's function be of a simple Lorentzian or delta-function form at low-temperatures [22,24]

$$
G_{0}(k) \simeq \frac{Z}{\xi^{-2}+\left(\vec{k}^{2}-q^{2}\right)^{2}}
$$

peaked about the manifold of minimizing modes $|\vec{k}|=q$ (Fig.(1)). As in [22], here $Z$ denotes the weight of the peak and $\xi$ the correlation length or inverse mass gap. For any unperturbed Green's function which may be written as a Laurent series in $k^{2}$, we may approximate the Green's function in the vicinity of its maximum by Eqn.(36). Eqn.(36) is the form analyzed long ago by Alexander and McTague [34] in the context of the liquid to solid transition. The bare Green's function $G_{0}$ is the approximate structure factor for a liquid with $l$ the average inter-atomic distance. If no cubic terms favoring crystallization are present in the action (i.e. if the transition to the crystal is "avoided"), and perform a self-consistent screening approximation (as done by [21]), then we will find a glass at low temperatures. An evaluation of the configurational entropy [22], [21]

$$
S_{c}=\int \frac{d^{3} k}{(2 \pi)^{3}}\left\{S\left[\frac{F}{G}\right]-S\left[\frac{F \otimes F}{(u T)^{-1}+G \otimes G}\right]\right\}
$$

with $S[x] \equiv-x-\ln (1-x)$ shows that the entropy is extensive within a broad temperature range $\left(T_{K}<T<\right.$ $T_{A}=T_{c}(q=0) /\left(q \pi^{2}+1\right)[21]$ and a vanishing configurational entropy density $S_{c}\left(T_{K}\right) / V=0$ defines the Kauzmann temperature $\left.T_{K}\right)$. In the above, $F$ is the EdwardsAnderson order parameter $\lim _{t \rightarrow \infty}\langle\phi(-k, t) \phi(k, 0)\rangle, G$ is the standard correlation function, and $\otimes$ denotes a convolution in Fourier space [22].

We now envision inverting the problem. Instead of starting from a certain fixed model and working our way through to find the configurational entropy, we go back to ask what sort of systems will exhibit extensive configurational entropy (i.e. an exponentially large number of metastable states) by the via provided by calculations that invoke the approximation of Eqn.(36). The answer is that the "universality class of systems" with Fourier transformed kernels $v(\vec{k})$ having their minima on the shells $|\vec{k}|=q>0$ ) and which are analytic in the vicinity of these minima, can have their Green's functions approximated by Eqn.(36) (e.g. non-Abelian background fields, myriad translationally invariant frustrated models with rotational symmetry). Once we assume this form, we may replicate the replica calculations of [22], [21] word for word to find that the configurational entropy is proportional to the volume $S_{c} \alpha V$, leading us to conclude that the system displays an exponentially large number of metastable states. The calculations of [21], [22] can be reproduced with slightly modified expressions for the onset temperature $T_{A}$ of glassy dynamics in various systems. Within all of these systems, including the dominant contribution from the lowest eigenvalue appearing in non-abelian gauge theories of glasses earlier, we find identical results. Repeating this calculations for temperatures $T<T_{A}$, the self-consistency conditions detailed in [21] are found to allow a nontrivial glassy state wherein defects may not, in all cases, venture more than $\mathcal{O}\left(q^{-1}\right)$ at arbitrarily long times.

For the $\mathrm{SO}(4)$ action of Eqn.(7), the lowest rank $n=12$ representation has $\tilde{N}=338$ individual real components. Within the large $\tilde{N} \gg 1$ limit, each individual complex eigenvector has a correlation length $\xi_{M, i}^{n}$ associated with it and the configurational entropy

$$
S_{c}=\sum_{M, i, n} S_{c}^{M, i, n}
$$

where $S_{c}^{M, i, n}$, the configurational entropy associated with each field $\tilde{\alpha}_{M, i}^{n}(x)$ (the Fourier transforms of the eigenmode amplitude $\left.\alpha_{M, i}^{n}(\vec{k})\right)$ is given by the self consistent 
screening approximation [21], [22] for a single scalar field (and carried in works till now by a large $\tilde{N}$ calculation, setting $\tilde{N}=1$ at its end). The field associated with the lowest eigenvalue, $\phi_{\min }$, has its minimizing wave-numbers on a finite radius, $q>0$, shell and its configurational $S_{c}^{\min }$ is extensive within the regime $T_{A}^{\min }>T>T_{K}^{\min }$. Trivially generalizing earlier calculations [21], the configurational entropy originating from the lowest eigenvalue,

$$
\begin{array}{r}
S_{c}^{\mathrm{min}}=\frac{q_{\mathrm{min}}^{3}}{4 \pi} V\left[\frac{\kappa_{\mathrm{min}}}{2}\left(1-\frac{\epsilon_{\mathrm{min}}}{\kappa_{\min }}\right)^{2}\right. \\
+\frac{2}{\pi}\left(\left(1-\frac{\epsilon_{\mathrm{min}}}{\kappa_{\mathrm{min}}}\right)^{2}+\ln \left(1-\left(1-\frac{\epsilon_{\mathrm{min}}}{\kappa_{\min }}\right)\right)\right] .
\end{array}
$$

Here [21], [22] $\kappa_{\min }$ satisfies

$\kappa_{\min }^{2}-\epsilon_{\min }^{2}=\frac{8 \epsilon_{\min }^{2}}{\pi} \frac{\left(1-\epsilon_{\min } / \kappa_{\min }\right)^{2}}{1-\left(1-\epsilon_{\min } / \kappa_{\min }\right)^{2}}\left(\frac{1}{\epsilon_{\min }}-\frac{1}{\kappa_{\min }}\right)$,

with $\epsilon_{\min }^{2}=\xi_{\min }^{-2} / q_{\min }^{4}$ and $V$ the volume. $S_{c}^{\min }\left(T_{c}\right)=$ $C V q_{\min }^{3}$, with the constant $C \simeq 1.18 \times 10^{-3} . T_{A, K}^{\min }$ may be for the single scalar field $\phi_{\min }[21],[22]$. As the configurational entropy is a sum over all eigenvalues, $S_{c}$ is generally extensive over a larger range than $T_{A, K}^{\min }$ suggest.

\section{SPATIALLY NON-UNIFORM SYSTEMS}

Thus far, we discussed ideal uniform frustration. We now illustrate that some of our ideas and calculations might not be so restricted. Any kernel $V_{i j}(\vec{x}, \vec{y})$ in the general two body Hamiltonian of Eqn.(3), becomes diagonal by some unitary transformation. The Fourier modes are the eigenmodes of $\hat{V}$ in the ideal translationally invariant setting. In general, $V_{i j}(\vec{x}, \vec{y})$ becomes diagonal in another complete orthogonal basis $(\{|\vec{u}\rangle\})$ :

$$
\left\langle\vec{u}_{\alpha}\left|V_{i j}\right| \vec{u}_{\beta}\right\rangle=\delta_{\alpha \beta}\left\langle\vec{u}_{\alpha}\left|V_{i j}\right| \vec{u}_{\beta}\right\rangle \equiv \delta_{\alpha \beta} v_{i j}\left(\vec{u}_{\alpha}\right) .
$$

We may now examine the minimizing manifold in $\vec{u}$ space for the lowest energy eigenvector. Repeating calculations, we find that if this surface if $(d-1)$ dimensional (e.g. a shell) and the relevant $v_{i j}(\vec{u})$ is quadratic in its environs then generally, for large $n, T_{c}=0$. The rigorous, large $n$, holographic ground state degeneracies of the previous section and the large $n$ configurational entropy analysis retain the same character. In many of the expressions above, the momentum index $\vec{k}$ is merely replaced by the more general diagonal basis index $\vec{u}$.

\section{GLASSY DYNAMICS}

In glasses, the relaxation times may increase by 15 orders of magnitude over a temperature range of $100 \mathrm{~K}$. It is impossible to obtain data below the glass transition temperature. All data has to be analyzed on a logarithmic scale. As a result, it hard by principle to make precise empirical statements in the vicinity of the "glass transition" itself (where the relaxation times, according to common wisdom, becomes infinite). We now discuss possible non-rigorous derivations for both the Vogel-Fulcher (VF) form (subsection A) and the avoided critical fit (subsection B). These two fits are different. There currently is no sufficient experimental or mathematical proof of any these forms. It is, ab initio, possible that both forms and their some of their assumed underlying theories are two aspects of the same problem. For instance, it is possible that the entropic-droplet/VF description is valid at very low temperatures whereas the avoided critical expression has its justification at higher temperatures (not far from the avoided critical temperature itself). Our calculations indicate that the theories of geometric frustration in glasses display both an avoided critical point and extensive configurational entropy. Albeit their suggestive character towards avoided critical and/or VF dynamics, none of these fits rigorously follows from the thermodynamics that we investigated.

\section{A. A Possible Derivation of Vogel-Fulcher Dynamics In Non-Abelian gauge backgrounds and others}

The basic premise of the VF fit is that the relaxation times seem to diverge at a temperature which correlates well with the intercept $\left(T_{K}\right)$ of the extrapolated entropy of the supercooled liquid with that of the solid. As outlined in the previous section, as the system is cooled down, the configurational entropy first becomes extensive $T_{A}$ (at which free energy minima first appear); at $T_{K}$ these minima become stable and the configurational entropy vanishes. Once we find an exponentially large number of metastable states for $T_{A}>T>T_{K}$ in nonAbelian backgrounds and other systems (by the replica calculations of the previous section), we may invoke the analysis of entropic droplets [25], [21]. This leads to characteristic energy barriers

$$
\Delta E \alpha\left(T S_{c}\right)^{-1} .
$$

Linearizing the extensive configurational entropy,

$$
S_{c}\left(T \rightarrow T_{K}^{+}\right) \sim V\left(T / T_{K}-1\right),
$$

this now leads to VF dynamics [25]. In the VF fit, the relaxation times

$$
\tau \sim \exp \left[D T_{K} /\left(T-T_{K}\right)\right]
$$

Here, $T_{K}$ is the Kauzmann temperature at (or above) which an "ideal glass transition" would occur. At $T_{K}$ the extrapolated entropy of the liquid undergoes a crisis.

Thus, once we show that the lowest eigenvalue of the non-abelian action has a minimum on a shell then we 
may non-rigorously derive VF dynamics. According to this line of logic, many of the translationally invariant systems with minimizing momenta $|\vec{k}|=q>0$ :

- Rigorously, do not order (at least in the usual sense) at finite temperature (the transition is narrowly avoided for $1 \gg q>0$ ).

- May display many of the characteristic thermodynamic signatures of narrowly avoided phase transition (e.g. a crossover of the thermodynamic functions at temperatures " $T_{1}$ " which merge with the critical temperature of the unfrustrated system in the limit of zero frustration (i.e. when $q \rightarrow 0)$ ).

- Rigorously, have numerous degenerate ground states, and, on a less rigorous footing, by an insertion of Eqn.(36) into Eqn.(37), have macroscopic configurational entropy (an exponentially large number of metastable states).

- The extensive configurational entropy of geometrically frustrated systems may lead to glassy (VogelFulcher like) dynamics at low temperatures. We may now non-rigorously derive VF dynamics by fusing standard approximations [25] with the extensive configurational entropy results of the replica calculations (Section(XI)).

\section{B. A Derivation of an Avoided Critical Point in the Avoided Critical Dynamics Ansatz}

As heralded in [12], perhaps nature follows another approach. One of the troubling (or surprising) aspects of the "glass transition" (if it is indeed a thermodynamic transition) is that although the dynamics undergoes a stupendous change- it may increase by orders of magnitude over a small temperature window- the smooth thermodynamic functions do not seem to know about any impinging transition. My coworkers and I [12] raised the possibility that the dynamics is governed by an avoided critical point. This viewpoint has two main assumptions:

1) The system would have undergone a phase transition in high enough dimensions but due to the frustration encountered in low dimensional Euclidean space it cannot. The phase transition is narrowly avoided leading to the phase diagram of Fig.(2).

2) The dynamics is tied at its core to the thermodynamics. The exponential increase in the relaxation times as the "glass transition" is approached is simply a reflection of a super-Arrhenius type dynamics $\tau \sim \exp (E / T)$ with the free energy barrier $E$ a function of the reduced temperature as measured relative to the avoided critical crystallization temperature on the unfrustrated high dimensional template (e.g. the 4-sphere on which icosahedral ordering can occur in the case of the simple monatomic system).

Here, we proved that in the large $n$ limit, assumption (1) for the geometrically frustrated actions introduced for glasses by many [1]. Our calculations yield the phase diagram of Fig.(2) for the $\tilde{N} \geq 338 \gg 1$ real component theories of glasses $\left(n \geq 12\right.$ in the $(n+1)^{2}$ complex components of the icosahedral multipole moments $\vec{Q}_{n}$ ). In Fig.(2), the ideal freezing temperature $T_{i f}=T_{c}(1 / R=0)$ is the transition temperature on the ideal template that is narrowly avoided in flat Euclidean space. This provides serious conformation to the notions underlying the avoided critical fit. We further proved, by a generalized Mermin-Wagner theorem, that no standard dispersion relations about nearly ordered states can exist at arbitrarily low temperatures for a host of systems, all of which display a minimum of the interaction kernel at finite wave-numbers compromising a high dimensional manifold in $\vec{k}$ space. Lately, numerical results [27] for clock realizations of a lattice version Hamiltonian of Eqn.(27), indeed show agreement with the form predicted for the scaling form of the free energy barrier [12] for this Coulomb frustrated model (Eqn.(44)). Furthermore, as we will now show, if we compare the magnitude of the avoided critical temperature $\left(T_{i f}\right)$ as inferred from the relaxational dynamics fit the free energy barrier, we will find that it agrees very well with what we would expect on simple energetic grounds. We provide further empirical impetus for assumption (2).

\section{Other Relaxational Forms}

Following this work, based on a new Monte Carlo analysis, [28] suggested that scalar systems with an on-shell momenta minimum display a pronounced slowing down yet not nearly as dramatic as that in true glasses. The dynamics is consistent with Hartree results. If true, when fused with the contents of this paper, this finding suggests that non-Abelian gauge background trigger a novel sluggish dynamics on their own right. This new dynamics, albeit very interesting, will have little to do with actual structural glasses. As detailed in later sections, structural glass theories must account for dynamics borne by topological defects (ignored in the uniform gauge background treatment). Within the relevant range of investigated parameters [29], a critical analysis of the data of [28] does not rule out glassy dynamics. It is possible that none of the forms presented (including the Hartree form) is correct for the continuum models of earlier sections. Nevertheless, all current simulations [27], [28] unambiguously point at sluggish dynamics implying very slow dynamics in non-Abelian backgrounds. Ingoring topolgoical defects all Landau-Ginzburg theories of liquids and crystals have a quadratic term with $v(\vec{k})$ having minima on the shell $|\vec{k}|=q$ (augmented by cubic terms in crystals and only quartic and higher in liquids) [34]. This suggests that if explicit topological defects are ignored then the most natural continuum actions for supercooled liquids are those which we investigate. 


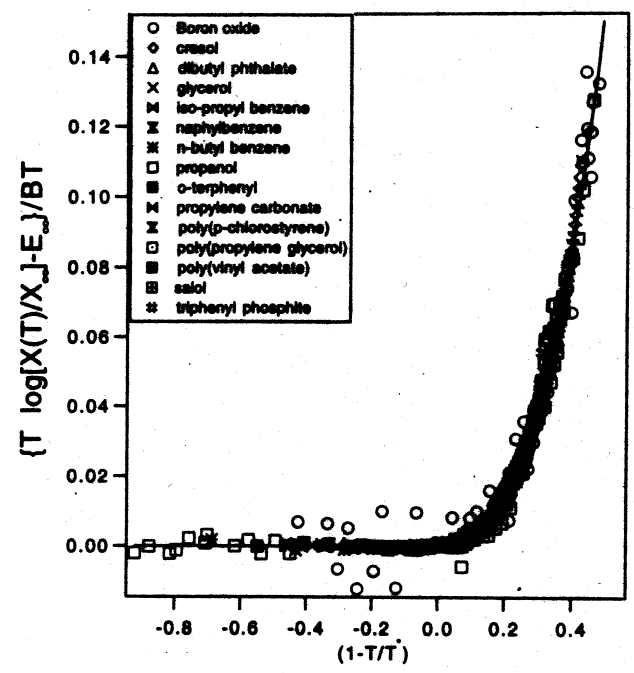

FIG. 6. This figure is from D. Kivelson et al. Here we fitted the relaxation times of all known glass formers to the super-Arrhenius form of Eqn.(44). The horizontal axis $\left(1-T / T^{i f}\right)$ gauges the reduced temperature as measured relative to the transition temperature $T^{i f}$ on the ideal curved template.

\section{PREDICTING $T_{I F}$ IN THE AVOIDED CRITICAL FIT FOR GLASSES}

We now examine the avoided critical dynamical fit of glasses [12] and present a comparison between the average avoided critical temperatures in these fits and the actual expected from simple energetic considerations (which seem to coincide remarkably well).

We saw that the simplest gauge theories of metallic glasses display an abrupt drop in their transition temperatures. The temperature of crystallization in 3 - dimensional Euclidean space is depressed well below the crystallization temperature on the curved ideal template. This discontinuity might be of profound importance: the thermodynamics (and consequently the dynamics) might be governed by the proximity to the "ideal freezing temperature" $T^{i f}$ - the temperature of crystallization on the ideal curved space template. In [12], we demonstrated that the following empirical fit works remarkably well for all known glass formers:

$\tau \simeq \tau_{\infty} \exp \left[E(T) /\left(k_{B} T\right)\right] ; E(T)=E_{\infty}+(a t)^{8 / 3} \theta(t)$

Here, $t \equiv\left(T^{i f}-T\right) / T^{i f}$, the relaxation time $\tau$ is deduced from viscosity and time dependent heat capacity measurements, and $\theta(t)$ is the Heavyside theta function.

We may expect $k_{B} T^{i f}$ to be indicative of the cohesive energy of the ideal curved space crystal, and the natural melting temperature scale $\left(k_{B} T_{\text {melt }}\right)$ to be correlated with the crystallization energy of the real (space) structure. Obviously entropic effects are important (e.g. those present in the $\mathrm{BCC} \rightarrow$ FCC transition [34]). Amongst these liquids, the average value of $x \equiv\left[T^{i f}-T_{m}\right] / T_{m}$ is $9.59 \%$. We may compare this to the value computed by Frank [26] for the relative cohesive energy, $\left(E^{i c o s a h d e r a l}-E^{F C C}\right) / E^{F C C}$, for a simple Lennard Jones liquid, which leads us to expect $\langle x\rangle=8.4 \%$. The proximity of these two values is misleading. $x$ varies dramatically from one liquid to another- the ideal crystalline structures, and their respective energies change radically from one material to another. Cautious optimism is still called for. If the theory were reliable and the parameter $T^{i f}$ obtained from the fit could indeed be interpreted as the freezing temperature on the ideal template, one would expect that, for all liquids, $x>0$, as the structure on the ideal template (where the ideal minimum energy local structure could be extended in all directions) is by its very definition more stable than (or at least as stable as) the corresponding structure constrained to reside in Euclidean space.

\begin{tabular}{|r|l|}
\hline \hline glass former & $x \equiv\left[T^{i f}-T_{m}\right] / T_{m}$ \\
\hline n-butyl benzene & $7.02 \%$ \\
triphenyl phosphite & $8.2 \%$ \\
isopropyl benzene & $20.6 \%$ \\
propylene carbonate & $7.73 \%$ \\
salol & $-4.4 \%$ \\
dibutyl phthalate & $21 \%$ \\
o-terphenyl & $5.7 \%$ \\
s-trinaphthyl benzene & $9.1 \%$ \\
n-propanol & $30.6 \%$ \\
$\alpha$-phenyl-cresol & $-10.3 \%$ \\
glycerol & $9.9 \%$ \\
\hline \hline glass former average & $9.59 \%$ \\
\hline \hline simple theory & $8.4 \%$ \\
\hline
\end{tabular}

\section{AVOIDED CRITICAL DYNAMICS AND MICROSCOPICS}

Ideal order cannot tessellate Euclidean space and it may do so only on a higher dimensional surface with energetics that seem to concur with the picture that we have in mind. In order to make progress toward understanding the dynamics we need to explicitly see how things transpire in real Euclidean space. We may adopt two trivial points of view: either the action is pinned to be that of a system in a fixed non-Abelian background, or the distribution of matter alters to the geometry by virtue of the topological defects that it carries in which case we have a full blown matter coupled gauge theory- an elastic theory with disclinations where the disclinations are not pinned down. The ideal order on the curved high dimensional template needs to be mapped into flat space by introducing the disclinations of Rivier to compensate for the lack of curvature in Euclidean space. 


\section{A. Fluctuating Geometry}

Let us assume that the ideal curved order is neutralized in flat Euclidean space by the insertion of a uniform disclination density (giving rise to an effective negative curvature to compensate for the positive curvature on the torn pieces of the ideal sphere). When glued together the curved pieces of the fractured sphere form disclinations at their common junctions. This is much like the seams that we encounter in orange peals that need to glued together somehow to form a flat surface. The disclination lines can easily be traced by looking at the locus of all points that have a lower or higher than usual coordination number as compared to that present in the ideal icosahedral tessellation. Formally, they can be seen by decurving processes relating the curved template to flat space. To a zeroth order approximation, the additional energy of the decurved structure (an elastic strain energy) amounts to the total length of the disclination network. In the context of decurving the $\{3,3,5\}$ polytope, the net number of disclinations is dictated by the number of sites having a coordination number different from the ideal (12-fold coordinated vertices). An analysis along this counting may be found in [1]. If we let the field $\phi$ denote the density of disclination lines then in our analysis such a zeroth order effect amounts to a chemical potential term $\mu \phi$ with the chemical potential $\mu$ now denoting the energy per unit disclination line.

Disclinations (or disclination pairs- dislocations) interact by direction dependent Coulombic like forces. In a two dimensional XY model like setting (e.g. vortices in a thin film superconductor) where the disclinations amount to additional twists that the phase must undergo when parallel transported about their cores. Such fractional vortices interact Coulombically. Two fractional vortices of opposite charges undo the damage that the other does at long distances. In three dimensions, the energy would be minimal for such wedge disclinations (or fractional vortices) lying in the same plane. In the tensorial elastic setting, such angle dependent forces undergo only simple detailed change. The Blin interaction between dislocations is a decorated Biot Savart interaction [32].

The same occurs in gravity. In $2+1$ dimensional gravity, masses amount to conical defects which cut and remove (or insert) additional angles in the plane much like disclinations. If we disregard "unimportant" details, trying to get to the heart of the matter by noting that the defect densities interact Coulombically, then we may immediately provide a possible derivation of the Coulomb frustrated Hamiltonian of Eqn.(27). We now try to give the reader a quick intuitive grasp of the microscopic derivation that what lacking till now (as still is). Let $\phi$ denote the defect density. In reality $\phi$ is a tensor by let's disregard details and merely see how things may work. For simplicity, we set the field $\phi$ be the local scalar curvature.
It is positive inside the broken fragments of the ideal sphere that have been flattened out; the net curvature is superposed on a neutralizing background that ensures that the net curvature is zero (as in the Frank-Kasper phases). Once we make the correspondence among the ideal sphere fragment densities and the disclination densities, eqn.(27) may, trivially, follow. The disclinations (or disclination pairs) interact Coulombically and the density toward ideal (high dimensional spherical) order within each resulting spherical fragment is captured by the gradient term. These two terms compete leading to extensive entropy at low temperatures and glassy dynamics. However, the dynamics is not trivially of a Vogel-Fulcher form.

To give the discussion a more detailed flavor we now give some of the standard expressions. Denoting by $\vec{u}$ the displacements of atoms from their ideal equilibrium positions, the dislocation density reads

$$
\alpha_{i j}=\epsilon_{i k l} \partial_{k} \partial_{l} u_{j}
$$

In a gravitational language, the dislocation density is, in simple disguise, the connection determining the change the of a vector due to parallel transport alone,

$$
\alpha_{i j}=\epsilon_{i k l} \Gamma_{k l j}
$$

with the connections

$$
\Gamma_{i j k}=\partial_{i} \partial_{j} u_{k}
$$

The connection between disclinations and gravitational curvature is even more transparent. With

$$
\omega_{i j}=\frac{1}{2}\left(\partial_{i} u_{j}-\partial_{j} u_{i}\right),
$$

the disclination density is

$$
\Theta_{i j}=\frac{1}{2} \epsilon_{i k l} \epsilon_{j q s} \partial_{k} \partial_{l} \omega_{q s}
$$

while the curvature tensor is

$$
R_{i j k l}=\left(\partial_{i} \partial_{j}-\partial_{j} \partial_{i}\right) \omega_{k l} .
$$

An explicit calculation for a wedge disclination corresponding to the insertion of an additional angle (of magnitude $\alpha$ ) in between the lips of a cut in the 12 plane shows that

$$
R_{1212}=-\alpha \delta^{2}\left(x^{\lambda}\right)
$$

where $\delta^{2}\left(x^{\lambda}\right)$ denotes the delta function in the plane for the disclination line $x^{\lambda}$ threading three dimensional space. Thus, the curvature tracks the disclination lines and its magnitude is proportional to the disclination angle. The disclination lines of Rivier [15] become mass sources. For a positive inserted angle, the scalar curvature is negative. If we wish to squash the angles to close 
the gaps such as those appearing when trying to piece together five tetrahedrons around a common edge. we insert a negative angle. The curvature, the full contraction of the curvature tensor,

$$
R=R_{\mu \nu}^{\mu \nu}
$$

is positive on a sphere (scaling as the inverse squared radius). The Blin interaction between dislocations

$$
E=\frac{\mu}{T} \int d^{3} x d^{3} x^{\prime} \alpha_{i j}(x)[K] \alpha_{l^{\prime} j^{\prime}},
$$

with the distance dependent part of the kernel $[K]$

$$
-\left(\frac{\epsilon_{l l^{\prime} k} \epsilon_{j j^{\prime} k}}{r}\right)+\frac{1}{1-\nu} \epsilon_{l j k} \epsilon_{l^{\prime} j^{\prime} k^{\prime}} \partial_{k} \partial_{k^{\prime}} r,
$$

where $\mu$ and $\nu$ are the elastic constants, and $\alpha$ the dislocation densities of Eqn.(45). All interactions between elastic defects are of a Coulombic character which has been tensorially flavored up. We can easily see why this must be so: Elasticity is centered around a second order gradient expansion,

$$
\begin{array}{r}
\mathcal{L}=\frac{1}{2} c_{i j k l} u_{i j} u_{k l}, \\
u_{i j} \simeq \frac{1}{2}\left(\partial_{i} u_{j}+\partial_{j} u_{i}\right),
\end{array}
$$

with $u$ the displacements about an equilibrium position. When Fourier transformed and inverted, the Green's functions through which the topological defects communicate always give rise to $k^{-2} \sim r^{2-d}$ interactions. In two dimensions, the interaction between the dislocations is, not too surprisingly, logarithmic. As all derivations that we have been able to devise (apart from the generalized Mermin-Wagner theorem and the construction of $O(n)$ ground states) have been approximate, we examine numerical results. Lately, computations [27] for clock realizations of a lattice version Hamiltonian of Eqn.(27), indeed show agreement with the form predicted for the scaling form of the free energy barrier [12] for this Coulomb model (Eqn.(44)). We have given simple arguments for the form of this fit, although a real theory is still lacking. As noted, the geometrical frustration breaks the glass into clusters. In an earlier publication [12], we have found that assuming the usual Ornstein-Zernicke form for $T<T_{c}$, within our "standard model", cumulant crossing occurs when the size of the domains is larger than $R_{D} \simeq \xi_{0}^{-1}$, with $\xi_{0}$ the correlation length of the unfrustrated system which diverges at the (avoided) critical temperature $T_{c}$. A somewhat similar result (with $\xi_{0}$ replaced by the correlation length $\xi$ of the frustrated system) followed from our $1 / \mathrm{n}$ calculation [11] for the Josephson like correlation length. This $1 / \mathrm{n}$ calculation result undergoes no change for any of the models that have their free energy minima on a shell in Fourier space which becomes sharp at low temperatures.
To shortly examine viable extensions later on, we sketch our derivation in [12]. Usually, the specific heat exponent $\alpha$ is small and consequently from the hyperscaling relation:

$$
\nu d=2-\alpha,
$$

we expect $\nu \simeq 2 / d$. The free energy barriers that govern the low temperature dynamics involve the flipping of domains (of size $R_{D}$ ). We assume that all is governed by the relative proximity to the avoided critical point. As in Eqn.(44), we consider temperatures below the avoided critical temperature $T<T^{i f}$ and denote by $t$ the absolute value of the relative reduced temperature. The correlation length in the unfrustrated system $\xi_{0} \sim t^{-\nu}$. The surface tension in the unfrustrated system

$$
\sigma \simeq B \frac{T_{i f}}{\xi_{0}^{d-1}},
$$

with $B$ a constant. The free energy barriers that we might find by breaking the medium into unfrustrated patches of size $R_{D}$ (the geometrical frustration in this approximation merely breaks the unfrustrated medium into finite patches (domains) of scale $R_{D}$ ),

$$
E \simeq \sigma R_{D}^{d-1} \simeq B T_{i f}\left(\frac{R_{D}}{\xi_{0}}\right)^{d-1}
$$

Employing our estimates [11,12] $R_{D} \sim \xi_{0}^{-1}$ and $\nu \sim 2 / d$,

$$
E \sim t^{4(d-1) / d}
$$

Substituting $d=3$ this gives an exponent of $8 / 3$ which which works best in our fit. It should be noted, however, that if we assume that

$$
E(T)=E_{\infty}+(a t)^{x}
$$

then for we can obtain reasonable fits with $7 / 3 \leq x \leq 3$.

\section{B. Fixed Non-Abelian Background}

Here no derivation of Eqn.(44) seems possible along the lines of the previous derivation. We may read off the dispersion from the lowest eigenvalue. For instance, in our toy model for the $\mathrm{SO}(3)$ action, $\lambda_{-}(k)=\mu+k^{2}+$ $3 \theta^{2} / 2-\frac{1}{2} \sqrt{16 k^{2} \theta^{2}+\theta^{4}}$ is the relevant dispersion. For $k \gg \theta$, (distances small compared to the zero temperature modulation length- the radius of the ideal sphere) the square root may be expanded and to lowest order, the dispersion is trivially $\left(k^{2}-2 k \theta+\mu\right)$. In a spin model, such a dispersion corresponds to a short range nearest neighbor ferromagnetic interaction $\left(k^{2}\right)$ frustrated by a longer range anti-ferromagnetic interaction $(-2 \theta|\vec{k}|)$. Unfortunately, the frustrating interaction is not of a much longer 
algebraic decay than the unperturbed ferromagnetic interaction. In a cumulant expansion similar to the one that we performed in [12], we now find that the characteristic domain size is $R_{D} \sim \xi_{0}^{2}$. When inserted back into our derivation for the free energy barriers this gives physically impossible dynamics: $E \sim t^{2(1-d) / d}$, a free energy barrier that quickly diminishes as we go away from the avoided critical point. For $k \ll \theta$ this worsens. We cannot see a natural generalization of the frustrated domains of the previous section which provides sensible results.

To summarize, for non-Abelian gauge backgrounds, we can very easily derive Vogel-Fulcher dynamics yet we are unable to derive avoided critical dynamics of the form of Eqn.(44). Notwithstanding, these models do display avoided critical behavior insofar as their thermodynamics is concerned. Furthermore, at low temperatures only the Fourier modes close to the minimizing shell should be of any importance. If a low temperature fit of the form of Eqn.(44) works well for the Coulomb frustrated Ferromagnet at small yet finite frustrations $e$, then Eqn.(44 might also capture the low temperature dynamics of all models (including the fixed non-Abelian background) that have their minima on a shell of finite radius in $k$ space. The approximate identification can be made by Taylor expanding the interaction kernels in the various cases to quadratic order and seeing that they all match.

\section{CONCLUSIONS}

To summarize, we have shown that a multitude of translationally invariant systems having their minimizing modes lie on a (d-1) dimensional shell in $\vec{k}$ space

1) Do not allow for a magnon dispersion relation about an ordered state at any finite temperature whenever a certain (Mermin-Wagner like) integral is seen to diverge.

2) We have noted that the generalized Mermin-Wagner inequality relates the magnetization (or its absence whenever entropy dominates) and the average of the expected relaxation times within the system.

3) The correlation and modulation lengths generally exhibit dynamics as a function of temperature (the imaginary time width). Crossovers in the asymptotic form of the correlation functions do, in general, occur at a finite temperature which veers toward the critical temperature of the unfrustrated system in the limit of zero frustration (zero curvature in myriad geometric models).

4) The systems exhibit an astonishing ground state and near ground state degeneracy. We may easily provide rigorous lower bounds on the ground state degeneracies and show, via replica calculations, that the configurational entropy is extensive. Physically, it is this multitude of degenerate and nearly degenerate states that makes the system so fragile to thermal perturbations. As a consequence, the ordering temperature the ordering temperature is very low.
5) We discussed how in a multitude of a systems (NonAbelian backgrounds and all others with an "on-shell" minima in Fourier space), the derivable extensive configurational entropy suggests Vogel-Fulcher like dynamics.

6) We illustrated how many of these notions can be extended to non-uniform system whereby the interactions are diagonal in a non-Fourier basis.

7) We have shown how the dynamics may indeed correlate with the avoided critical fit of [12] by comparing the melting temperatures with the fitted temperatures. The average values are in good agreement with our theory.

8) We suggested a via for strengthening the link between avoided critical dynamics [12] with microscopic consideration regarding interactions between disclinations.

\section{ACKNOWLEDGMENTS}

Most of this work was covered in my thesis long ago [24]. During my thesis years I had the good fortune of working with many good friends. A few deserve close mention- S. A. Kivelson, J. Rudnick, D. Kivelson, G. Tarjus, and L. Chayes. I also wish to thank D. Nógrádi and J. Sethna for a discussion.

\section{APPENDIX A: $N \rightarrow \infty$ AVOIDED CRITICALITY IN THE QUANTUM ARENA}

The single $n \rightarrow \infty$ parquet diagram (Fig.(4)) reads

$$
\Sigma^{0}=\frac{n u T}{2} \sum_{\omega_{m}} \int \frac{d^{d} k}{(2 \pi)^{d}} \frac{1}{\omega_{m}^{2}+v(\vec{k})+\mu_{m i n}}
$$

with the bosonic Matsubara frequencies $\omega_{m}=2 \pi m T$.

$\Sigma^{0}=\frac{n u T}{2}\left(\frac{1}{2 \pi T}\right)^{2} \sum_{m} \int \frac{d^{d} k}{(2 \pi)^{d}}\left[m^{2}+\left(\omega_{\vec{k}} /(2 \pi T)\right)^{2}\right]^{-1}$

where $\omega_{\vec{k}}=\sqrt{\mu+v(\vec{k})}$. The sum can be evaluated by the method of residues which gives

$$
\sum_{m=-\infty}^{\infty}\left[m^{2}+y^{2}\right]^{-1}=\frac{\pi}{y} \operatorname{coth} \pi y \quad \text { for } y>0 .
$$

Using this we obtain

$$
\Sigma^{0}=\frac{1}{4} \int \frac{d^{d} k}{(2 \pi)^{d}} \frac{1}{\omega_{\vec{k}}} \operatorname{coth}\left(\frac{\omega_{\vec{k}}}{2 T}\right) .
$$

From the identity

$$
\operatorname{coth}\left(\frac{x}{T}\right)=1+2 n_{B}\left(\frac{2 x}{T}\right)
$$

where $n_{B}\left(\frac{x}{T}\right)$ represents the bosonic distribution function 


$$
n_{B}\left(\frac{x}{T}\right)=\left[\exp \left(\frac{x}{T}\right)-1\right]^{-1}
$$

Thus

$$
\Sigma^{0}=\frac{n u}{4} \int \frac{d^{d} k}{(2 \pi)^{d}} \frac{2 n_{B}\left(\frac{\sqrt{v(\vec{k})+\mu}}{T}\right)+1}{\sqrt{v(\vec{k})+\mu}} .
$$

As $\mu \rightarrow \mu_{\min }=-v(\vec{q})$, this integral diverges more strongly than in the classical case. This divergence remains down to zero temperature. The integrand is (up to a constant) the static correlation function.

$$
G(\vec{k})=\frac{1}{2} \frac{2 n_{B}\left(\frac{\sqrt{v(\vec{k})+\mu}}{T}\right)+1}{\sqrt{v(\vec{k})+\mu}} .
$$

\section{APPENDIX B: THE SO(4) SOCCER-BALL MODEL ON A CUBIC LATTICE}

For $O(4)$ spins with $S O(4)$ couplings in $d=3$ :

$$
D_{\nu} S^{\mu}=\partial_{\nu} S^{\mu}+S^{\lambda} \Gamma_{\nu \lambda}^{\mu}
$$

For four-component spins lying on a three-dimensional lattice (i.e. when the continuum limit is not taken) having $S O(4)$ couplings " $[-\vec{S}(\vec{x}) * \vec{S}(\vec{y})]$ " the matrix $-v_{i, j}(\vec{k}) / 2$ reads

$$
\left(\begin{array}{cccc}
A & 0 & 0 & -E \\
0 & B & 0 & -F \\
0 & 0 & C & -G \\
E & F & G & D
\end{array}\right)
$$

where

$$
\begin{array}{r}
A=\cos \theta \cos k_{1}+\left(\cos k_{2}+\cos k_{3}\right) \\
B=\left(\cos k_{1}+\cos k_{3}\right)+\cos \theta \cos k_{2} \\
C=\sum_{l=1}^{2} \cos k_{l}+\cos \theta \cos k_{3} \\
D=\cos \theta \sum_{l=1}^{3} \cos k_{l} \\
E=i \sin \theta \sin k_{1} \\
F=i \sin \theta \sin k_{2} \\
G=i \sin \theta \sin k_{3}
\end{array}
$$

Note the appearance of a non-uniform sign (and nontrivial phases) linking the various spin components. These play the role of frustrating interactions present in scalar theories; in a high temperature expansion for the partition function $Z$, not all terms are the sum of different contributions, all adding in unison with the same phase (and therefore also sign). The alternating signs of the resulting constituent terms are analogous to those generated in other frustrated systems by competing ferromagnetic and antiferromagnetic interactions in the evaluation of the partition function $Z$. For a nonzero value of the angle $\theta, T_{c}(\theta \neq 0)=0$. The minimizing modes lie on a $(d-1)$ dimensional shell.

\section{APPENDIX C: EXPLICIT EXPRESSIONS FOR CORRELATION FUNCTION CROSSOVERS}

\section{Uniform $\mathrm{SO}(3)$ and $\mathrm{SO}(4)$ backgrounds}

For the $S O(3)$ action of [7] in $\mathrm{d}=2$ dimensions, summing over a set of $(2 \ell+1) \times(2 \ell+1)$ representations with corresponding rank spherical tensor order parameters:

$$
H=\frac{1}{2} \sum_{\ell} \int d^{2} x\left[K_{\ell}\left|\left(\partial_{\mu}+\imath \theta \epsilon_{\mu \nu} \hat{L}_{\nu}^{(\ell)}\right) \vec{\phi}_{\ell}\right|^{2}+\mu_{\ell}\left|\overrightarrow{\phi_{\ell}}\right|^{2}\right]
$$

with $\left\{\mu_{\text {ell }}\right\}$ chemical potentials- Lagrange multipliers- set to secure overall normalization of $\left\{\vec{\phi}_{\ell}\right\}$. In $\vec{k}$-space,

$$
\begin{array}{r}
H=\frac{1}{2 N} \sum_{\ell} \sum_{i} \sum_{\vec{k}}\left|\alpha_{\ell}^{i}(\vec{k})\right|^{2}\left[K_{\ell} \lambda_{\ell}^{i}(\vec{k})+\mu_{\ell}\right] \\
\text { with } \phi_{\ell m}(\vec{k})=\sum_{i=1}^{2 \ell+1} \alpha_{\ell}^{i}(\vec{k}) e_{\ell m}^{i}(\vec{k}) .
\end{array}
$$

The self-consistent equation of constraint that the Lagrange multipliers must satisfy is

$$
\frac{1}{k_{B} T}=\int \frac{d^{2} k}{(2 \pi)^{2}} G(\vec{k})
$$

The inverse critical temperature, $\frac{1}{k_{B} T_{c}}$, diverges as the reciprocal of a lower cutoff on $|k-q|$. We find that $(2 \ell+$ 1) poles are generically present for each $\ell$ when Fourier transforming

$$
G(\vec{k})=\sum_{\ell} \frac{2 \ell+1}{4 \pi} \sum_{i}\left\langle\alpha_{\ell}^{i}(\vec{k}) \alpha_{\ell}^{j}(-\vec{k})\right\rangle e_{\ell 0}^{i}(\vec{k}) e_{\ell 0}^{j}(-\vec{k}),
$$

which simplifies to

$$
G(\vec{k})=\sum_{\ell} \frac{2 \ell+1}{4 \pi} \sum_{i=1}^{2 \ell+1} \frac{k_{B} T}{K_{\ell} \lambda_{\ell}^{i}(\vec{k})+\mu_{\ell}}\left|e_{\ell, 0}^{i}(\vec{k})\right|^{2} .
$$

Our two-dimensional soccer-ball $(\ell=1)$ Hamiltonian:

$$
H=-\sum_{\langle\vec{x}, \vec{y}\rangle} \vec{S}(\vec{x}) * \vec{S}(\vec{y})
$$


leads to three poles at all temperatures. Much unlike the pole locations, the branch points are temperature independent. If we ignore the $\vec{k}$ dependence of $\left|e_{1,0}^{ \pm}(\vec{k})\right|^{2}$, then the contribution to $G(|\vec{x}|)$ from the branch cut contour will read

$$
\frac{\theta}{4 \pi x^{2}}\left(A_{+}-A_{-}\right) \int_{\theta / 4}^{\infty} d v \frac{v \sqrt{\theta^{2}-16 v^{2} / x^{2}} I_{0}(v)}{\left(\mu-\frac{v^{2}}{x^{2}}+\frac{3}{2} \theta^{2}\right)^{2}-\theta^{2}\left(\theta^{2}-16 \frac{v^{2}}{x^{2}}\right)}
$$

where $A_{ \pm}=\frac{3}{4 \pi}\left|e_{1,0}^{ \pm}\right|^{2} k_{B} T$ and $I_{0}$ a Bessel function of imaginary argument. The contributions from the branch cut would seem (if $\left\{e_{\ell=1,0}^{ \pm}\right\}$were $\vec{k}$ independent) to give rise to quasi-algebraically damped correlations.

For the case at hand,

$$
\begin{gathered}
\left|u_{-}\right\rangle=N_{-}\left|\frac{4 i \theta k_{y}}{\theta^{2}-\sqrt{16 k^{2} \theta^{2}+\theta^{4}}}, \frac{4 i \theta k_{x}}{\theta^{2}-\sqrt{16 k^{2} \theta^{2}+\theta^{4}}},-1\right\rangle, \\
\left|u_{+}\right\rangle=N_{+}\left|\frac{4 i \theta k_{y}}{\theta^{2}+\sqrt{16 k^{2} \theta^{2}+\theta^{4}}}, \frac{4 i \theta k_{x}}{\theta^{2}+\sqrt{16 k^{2} \theta^{2}+\theta^{4}}}, 1\right\rangle,
\end{gathered}
$$

are the eigenvectors expressed in the Cartesian basis. In the spherical tensor basis $\left\{\left|e_{ \pm}^{1,0}\right|^{2}\right\}$ are the squared moduli $\left|N_{ \pm}\right|^{2}$, of the z-components of the eigenvectors $\left|u_{ \pm}\right\rangle$are

$$
\left|e_{\ell=1,0}^{ \pm}\right|^{2}=1-\frac{8 k^{2} \theta^{2}}{\theta^{4} \pm \theta^{3} \sqrt{16 k^{2}+\theta^{2}}+16 \theta^{2} k^{2}} .
$$

The dependence of $\left|e_{\ell=1,0}^{ \pm}\right|^{2}$ on $|\vec{k}|$ is manifest. Inserting this in $G(\vec{k})$ and Fourier transforming one readily verifies that no quasi-algebraic behavior remains only the usual exponential correlations originating from simple poles appear. For infinitesimal curvature (or frustrations) $\theta$, all correlation lengths (originating from these poles $\left|\operatorname{Im}\left\{k_{o}\right\}\right|^{-1},\left|\operatorname{Im}\left\{k_{ \pm}\right\}\right|^{-1}$ can be made as large as desired when $T \rightarrow 0: k_{o}^{2}=-\left[\mu+\theta^{2}\right], k_{ \pm}^{2}=\frac{1}{2}\left[\theta^{2}-2 \mu \pm\right.$ $\left.i \sqrt{16 \mu \theta^{2}+7 \theta^{4}}\right]$. Note that here there is no crossover of a correlation length into a modulation length: The poles $\left\{ \pm k_{ \pm}^{2}\right\}$ are complex conjugate for $\mu>\mu_{\min }=-7 \theta^{2} / 16$ and will give to a damped oscillatory behavior at all temperatures. Nonetheless, a crossover does occur (albeit not a thermodynamic one). At very low temperatures the long correlations are spawned by $k_{ \pm}$and are are therefore oscillatory. At intermediate temperatures the correlation length generated by $k_{0}$ can be larger leading to asymptotic long range correlations that are non-oscillatory in character. To see this, we note that

$$
\begin{array}{r}
\left|\operatorname{Im}\left\{k_{0}\right\}\right|=\sqrt{\mu} \\
\left|\operatorname{Im}\left\{k_{ \pm}\right\}\right|=\left(2 \theta^{4}+3 \mu \theta^{2}+\mu^{2}\right)^{1 / 4}|\sin (\phi / 2)| \\
\phi=\tan ^{-1}\left(\frac{\sqrt{16 \mu \theta^{2}+\theta^{4}}}{\theta^{2}-2 \mu}\right) .
\end{array}
$$

Whenever $\mu(T)=\theta^{2} / 2$,

$$
\begin{array}{r}
\left|\operatorname{Im}\left\{k_{0}\right\}\right|=\frac{\theta}{\sqrt{2}} \\
\left|\operatorname{Im}\left\{k_{ \pm}\right\}\right|=(3.75)^{1 / 4}\left|\operatorname{Im}\left\{k_{0}\right\}\right| .
\end{array}
$$

At this temperature $\left|\operatorname{Im}\left\{k_{ \pm}\right\}\right|>\left|\operatorname{Im}\left\{k_{0}\right\}\right|$ and therefore the asymptotic correlations will not be oscillatory as they are near the ground state. In this way we can identify a low temperature crossover. It is straightforward to obtain a simple expression for the value of the chemical potential $\mu$ at this crossover temperature.

$$
\begin{array}{r}
\left|\operatorname{Im}\left\{k_{ \pm}\right\}\right|=2^{-1 / 2}\left(2 \theta^{4}+3 \mu \theta^{2}+\mu^{2}\right)^{1 / 4} \\
\times \sqrt{1-\left[1+\frac{16 \mu \theta^{2}+\theta^{4}}{\left(\theta^{2}-2 \mu\right)^{2}}\right]^{-1 / 2}} \\
=\left|\operatorname{Im}\left\{k_{0}\right\}\right|=\sqrt{\mu} .
\end{array}
$$

For larger matrices (e.g. $\ell>1$ in an $S O(3)$ action) there are potentially more crossovers. For these matrices, at asymptotically large temperatures all correlation lengths $\rightarrow \mu^{-1 / 2}$. As we will see, this is not the case for long range interactions Notice that this change from a uniform behavior at high $T$ to finite modulation lengths at low $T$ is a feature shared in common with the Coulomb frustrated ferromagnet (and any other system with a frustrating long range interaction). We find similar results for the $S O(4)$ action in three dimensions of [7]. The correlator

$$
G(\vec{k})=\sum_{n} \frac{n+1}{2 \pi^{2}} \frac{k_{B} T}{K_{n} \lambda_{p=0, i}^{n}(|\vec{k}|)+\mu_{n}}\left|\sum_{m} e_{n, m m}^{i}(\vec{k})\right|^{2},
$$

with $i$ a single index labeling the eigenvalues, where we note the commutativity of $\left[M_{+}-M_{-}\right]$with the kernel.

\section{Correlation Function Crossovers Within the Coulomb Frustrated Ferromagnet}

Unlike other Appendices, most of the contents of this section appear in a Letter version of our work on Coulomb frustration [11]. The continuum limit Hamiltonian of the Coulomb frustrated ferromagnet

$$
H=\frac{1}{2} \int \frac{d^{d} k}{(2 \pi)^{d}}\left(k^{2}+e^{2} k^{-2}+\mu\right)|S(\vec{k})|^{2}
$$

where the spherical constraint (global spin normalization) is enforced via the chemical potential $\mu$. A nonincreasing weight for all modes $\vec{k}$ mandates $\mu \geq-2 e$. The minimizing modes $\vec{q}^{2}=e$ ). By equipartition, the mode occupancy $\left\langle|S(\vec{k})|^{2}\right\rangle=k_{B} T /\left(k^{2}+e^{2} / k^{2}+\mu\right)$. The correlator:

$$
\begin{aligned}
G(\vec{x}) & =\frac{1}{(2 \pi)^{3}} \int d^{3} k\left\langle|S(\vec{k})|^{2}\right\rangle \exp [i \vec{k} \cdot \vec{x}] \\
& =\frac{k_{B} T}{2 \pi^{2}|\vec{x}|} \int_{0}^{\infty} d k \frac{k^{3}\left[\operatorname{Im}\left\{e^{i k|\vec{x}|}\right\}\right]}{\left(k^{2}+\alpha^{2}\right)\left(k^{2}+\beta^{2}\right)}
\end{aligned}
$$


where

$$
\alpha^{2}, \beta^{2}=\frac{\mu \mp \sqrt{\mu^{2}-4 e^{2}}}{2} .
$$

When $\mu>2 e$, the integral can be readily evaluated by applying the residue theorem to the poles lying on the imaginary axis at $k= \pm \imath \alpha, \pm \imath \beta$,

$$
G(\vec{x})=\frac{k_{B} T\left(\beta^{2} e^{-\beta|\vec{x}|}-\alpha^{2} e^{-\alpha|\vec{x}|}\right)}{4 \pi|\vec{x}|\left(\beta^{2}-\alpha^{2}\right)} .
$$

Note the existence of two macroscopic correlation lengths - a consequence of charge neutrality. As the spins portray charges, they must sum to zero,

$$
\int G(\vec{x}) d^{3} x=\left\langle\left|\int S(\vec{x}) d^{3} x\right|^{2}\right\rangle=0 .
$$

Whenever $G$ is dominated by its long-distance behavior, the integral can vanish only if $G(x)$ contains positive and negative contributions, as in Eqn.(C16). This integral can vanish only if $G(\vec{x})$ contains, at least, two length scales. At high temperatures, the length

$$
l_{1} \equiv|\operatorname{Re}\{\beta\}|^{-1} \approx \mu^{-1 / 2}(\text { for } \mu \gg 2 e) .
$$

plays the role of the correlation length of the canonical short-range ferromagnet (i.e. with no frustrating charge$e=0)$. Note that now, however, an additional correlation length appears:

$$
l_{2} \equiv|\operatorname{Re}\{\alpha\}|^{-1} \approx \frac{1}{\xi_{1} e} .
$$

For $\mu^{2}=4 e^{2}$, we find that $\alpha=\beta$, and the four poles merge in pairs. When $\mu^{2}<4 e^{2}$ the poles reside on a circle of radius $e^{1 / 4} ; \operatorname{Re}\left\{\alpha^{2}\right\}=\operatorname{Re}\left\{\beta^{2}\right\}=\frac{r}{2}$. This corresponds to a pole at $k$ on the circle of radius $e^{1 / 2}$ at an angle $\theta$ with $\cos 2 \theta=-r /(2 e)$. The analytic continuation for $\mu^{2}<4 e^{2}$ is trivially $\left(\alpha \equiv \alpha_{1}+\imath \alpha_{2}=\beta^{*}\right)$ :

$$
\begin{aligned}
G(\vec{x})= & \frac{k_{B} T}{4 \pi} \exp \left(-\alpha_{1}|\vec{x}|\right) \\
& \times\left[\frac{\left(\alpha_{2}^{2}-\alpha_{1}^{2}\right) \sin \alpha_{2}|\vec{x}|+2 \alpha_{1} \alpha_{2} \cos \alpha_{2}|\vec{x}|}{4 \alpha_{1} \alpha_{2}|\vec{x}|}\right]
\end{aligned}
$$

where $\alpha \equiv \alpha_{1}+\imath \alpha_{2}$.

Putting all of the pieces together:

$$
\text { for } \begin{array}{r}
T>T_{1} \equiv k_{B}^{-1}(2 \pi)^{3} /\left[\int^{\Lambda} d^{3} k /\left(2 e+\frac{e^{2}}{k^{2}}+k^{2}\right)\right] \\
>T_{c}(e=0) ; \ell_{1} \neq \ell_{2},
\end{array}
$$

$$
\text { for } T<T_{1} \quad \ell_{1}=\ell_{2} \text {. }
$$

The temperature $T_{1}$, defined by $\mu\left(T=T_{1}\right)=2 e$, marks a dramatic crossover. At low temperatures $(T<$ $T_{1}$ ), the system possesses a single correlation length $\xi=$ $\left|\alpha_{1}\right|^{-1}=2[\mu+2 e]^{-1 / 2}$, and a single modulation length $L_{D}=2 \pi /\left|\alpha_{2}\right|=4 \pi[-\mu+2 e]^{-1 / 2}$; at high temperatures $\left(T>T_{1}\right)$, the system possesses two distinct correlation lengths. When $T=T_{1}^{-}$, the modulation length diverges as $L_{D} \sim\left(T_{1}-T\right)^{-1 / 2}$. The crossover temperature and the critical temperature read

$$
\begin{array}{r}
T_{1} \simeq 2 \pi^{2}\left[\Lambda-\frac{3 \pi}{4} e^{1 / 2}\right]^{-1}, \\
T_{c}(e=0)=\frac{2 \pi^{2}}{\Lambda},
\end{array}
$$

with $\Lambda$ an upper momentum cutoff.

The mechanically oriented reader might note a simple analogy to the temporal quadratic form for the damped oscillator, where two relaxation times appear for $\gamma^{2}>$ $4 m \kappa$ (with $\gamma, \kappa$, and $m$ the dissipation coefficient, the spring constant and the mass respectively). At high temperatures, $\ell_{1} \approx \mu^{-1 / 2}$ plays the role of the usual correlation length (for a free Landau-Ginzburg action with the quadratic kernel $\left.v(\vec{k})=\mu+k^{2}\right)$; this is the analogue of the damping time in an "un-driven" mechanical system. At low temperatures $\left(T<T_{1}\right)$, the information previously encoded in two correlation lengths at high temperatures is now manifest as a single correlation length $\ell=\left|\alpha_{1}\right|^{-1}=2 \sqrt{\frac{1}{2 e+\mu}}$ and a single modulation length: $L_{D}=2 \pi /\left|\alpha_{2}\right|=4 \pi \sqrt{\frac{1}{2 e-\mu}}$. The "domain size" $L_{D}$, the inverse characteristic sinusoidal modulation length of the correlations, diverges at $T=T_{1}$, the correlation length at $T_{c}=0$. The value registered by $\mu$ at the avoided critical temperature $T_{c}(e=0)$ is roughly dimension independent and pinned at $\mu \approx-e$; both $L_{D}$ and $\ell$ are regular at $T_{c}(e=0)$. Note that, $\lim _{e \rightarrow 0+} T_{1}(e)=T_{c}(e=0)$ (this statement can be well defined only in a slightly modified model such as $v(\vec{k})=k^{2}+e^{2} k^{-2}+\lambda \sum_{i \neq j} k_{i}^{2} k_{j}^{2}$ with small $\lambda$ ). In that limit $L_{D}$ makes an entrance at the "avoided critical point" $T_{c}(e=0)$. Such a crossover of a correlation length(s) into a modulation length(s), or vice-versa, is a feature that is generically absent in finite Range interaction systems.

\section{Correlation Functions for Other Short Range Frustrated Models and others}

Consider the interaction with the kernel

$$
v(\vec{k})=\left[\Delta^{3}-\Delta_{0}^{3}\right]^{2},
$$

where the lattice Laplacian

$$
\Delta(\vec{k})=2 \sum_{l=1}^{d}\left(1-\cos k_{l}\right),
$$

with $\left\{k_{l}\right\}_{l=1}^{d}$ the momentum components. As the lattice Laplacian connects, in real space, two sites that 
are a lattice distance apart, the kernel of Eqn.(C24) may link sites that are, at most, six lattice constants apart. It corresponds to a Range $=6$ interaction. If the constant $\Delta_{0}>0$ then upon expanding Eqn.(C24), we will find both positive and negative contributions. Expanding Eqn.(C24), we find both ferromagnetic and anti-ferromagnetic interactions that compete on different ranges. When a chemical potential term is added to enforce normalization, we find that its minimum value is $\mu_{\min }=\Delta_{0}^{6}$ (whence the mass gap vanishes). For future reference let us denote the mass gap by $c \equiv\left(\mu-\mu_{\min }\right)$. The poles of the propagator

$$
G(\vec{k})=k_{B} T[c+v(\vec{k})]^{-1}
$$

occur at

$$
\left\{\Delta_{\alpha}^{ \pm}\right\}_{\alpha=1}^{3}=\left[\Delta_{0}^{3} \pm \imath \sqrt{c}\right]^{1 / 3} \exp (2 \pi \alpha \imath / 3) .
$$

The poles $\left\{\Delta_{\alpha}^{ \pm}\right\}$form the vertices of a hexagon in the complex $k$ plane. Whenever $k_{i}^{3}=k_{o}^{3}$ with $k_{o}$ a given complex number, then there must exist, at least, two roots with different imaginary values $\left(\left|\operatorname{Im}\left\{k_{i}\right\}\right|\right)$. The correlation lengths $\ell_{i}=\left|\operatorname{Im}\left\{k_{i}\right\}\right|^{-1}$. Two different correlation lengths $\ell_{1} \neq \ell_{2}$ can be identified at all $\mu \geq \mu_{\text {min }}$ $\left(\mu=\mu_{\min }\right.$ only at $\left.T=T_{c}=0\right)$. We note the existence of multiple correlation lengths $\ell_{i}$ at all values of $\Delta_{0}$ and temperatures (the mass gap $c$ ). Multiple correlations lengths are also present everywhere- this also includes the unfrustrated system having $\Delta_{0}<0$ ! Multiple correlation lengths are generically present for functions of $\Delta$ (or $k^{2}$ in the continuum) and are accompanied by a discontinuity in the critical temperature $T_{c}$ in sufficiently high dimension whenever appropriate competition allows real roots $0<\left\{\Delta_{i}\right\}<4 d$ when the mass gap vanishes $\left(\mu=\mu_{\min }\right)$. For the canonical $\Delta$ - polynomial, the system possesses several modulation lengths and several correlation lengths at all $\mathrm{T}$; barring few exceptions - their net number is conserved as temperature is varied. Generically, all cross-over temperatures $\left[T_{i=1,2, \ldots, p}\right]$ (including low dimensional systems which possess no critical behavior for zero frustration), at which correlation lengths disappear and turn into modulation lengths, tend continuously to the avoided critical temperature (or its analytic continuation for low dimensions - in high dimensions this temperature $\mu(T)=\mu^{*}$ becomes critical for zero frustration). For a general finite ranged $\Delta$ polynomial kernel, the system possesses a fixed number of correlation lengths and a fixed number of modulation lengths; there is no sharp analogue of crossover temperatures $\left\{T_{i}\right\}$ wherein modulation lengths turn into correlation lengths. These crossover temperatures are more prevalent for non-analytic functions of $\Delta$. The Yukawa Ferromagnet $v(\vec{k})=\mu+\left(k^{2}+\lambda^{2}\right)+\frac{e^{2}}{k^{2}+\lambda^{2}}$ (in real space the last term gives rise to Yukawa like screened interactions) has $T_{c}\left(e=\lambda^{2}\right)>0$ in $d>4$ and in any dimension $T_{c}\left(e>\lambda^{2}\right)=0$. Two correlation lengths appear for $\mu^{2}>4 e^{2}$ (including all unfrustrated ferromagnets $(e<0))$.

For the non-analytic kernel $v(\vec{k})=(|\vec{k}|-q)^{2}$,

$$
\begin{aligned}
& \frac{1}{k_{B} T} \simeq \frac{1}{2 \pi^{2}}\left[\Lambda+\frac{q^{2}-\mu}{\sqrt{\mu}}\left\{\tan ^{-1}\left(\frac{\Lambda-q}{\sqrt{\mu}}\right)\right.\right. \\
& \left.\left.+\tan ^{-1}(q / \sqrt{\mu})\right\}+q \ln \left(\frac{\mu+(\Lambda-q)^{2}}{\mu+q^{2}}\right)\right],
\end{aligned}
$$

with $\mu_{\min }=0$.

The correlator corresponding to $v(\vec{k})=(|\vec{k}|-q)^{2}$,

$$
\begin{array}{r}
G(\vec{x})=\frac{1}{2 \sqrt{\mu}|\vec{x}|} e^{-\sqrt{\mu}|\vec{x}|}[q \sin q x+\sqrt{\mu} \cos q x]- \\
\frac{q I(|\vec{x}|)}{\pi^{2}|\vec{x}|},
\end{array}
$$

where

$$
I(|\vec{x}|)=\int_{0}^{\infty} d \kappa\left[\frac{\kappa \exp [-\kappa|\vec{x}|]}{\left(\mu+q^{2}-\kappa^{2}\right)^{2}+4 q^{2} \kappa^{2}}\right]>0 .
$$

Note the algebraic decay in this artificial non-analytic system. Here the system displays avoided critical behavior and exhibits one (two) modulation lengths $2 \pi / q$ (and infinity), which does not vary with temperature. Within the spherical model, this behavior is an exception: modulation lengths tend to vary with temperature.

With $\left.t \equiv\left(k_{B} T_{c}(q=0)\right)^{-1}-\left(k_{B} T\right)^{-1}\right)$, we find at low temperatures $\left(\mu^{3 / 2}\right.$ terms neglected $)$ for the $v(\vec{k})=$ $(|\vec{k}|-q)^{2}$ action,

$$
\begin{array}{r}
t \approx\left(1+\frac{1}{2 \pi^{2}} \frac{q}{\Lambda-q}\right) \mu+\frac{1}{2 \pi} \sqrt{\mu} \\
-\frac{1}{2 \pi^{2}} q \ln \left(\frac{\mu+(\Lambda-q)^{2}}{\mu+q^{2}}\right)-\frac{1}{2 \pi} \frac{q^{2}}{\sqrt{\mu}} .
\end{array}
$$

In the low temperature $\mu \rightarrow 0^{+}$limit: $t \simeq-\frac{1}{2 \pi} \frac{q^{2}}{\sqrt{\mu}}$ (as in any dimension $d \neq 3$ apart from a constant multiplicative factor). The large $n$ critical exponent $\nu_{q>0}=1$. At very low temperatures $(t<0$ with large $|t|)$,

$$
\sqrt{r} \sim q^{2} /|t| \sim q^{2}\left[\ell_{q=0}(-t)\right] .
$$

If, by analogy to the standard quadratic part of LandauGinzburg actions, we define a length $L_{D} \sim r^{-1 / 2}$, the inverse square root of the mass gap which is the correlation length in the canonical $(q=0)$ case, then as $T \rightarrow 0^{+}, L_{D} \sim e^{-1} \ell_{q=0}^{-1}\left(\right.$ with $\left.q=e^{1 / 2}\right)$. We point here to the strong resemblance to the previously obtained result for the exact correlation lengths appearing for the Coulomb frustrated ferromagnet with $v(\vec{k})=\left[e^{2} / k^{2}+k^{2}\right]$ at high temperatures.

For the short-range (Teubner-Strey) correlator

$$
G^{-1}(\vec{k})=a_{2}+c_{1} k^{2}+c_{2} k^{4},
$$


it is a simple matter to show that

$$
G(\vec{x}) \sim \frac{\sin \kappa x}{\kappa x} \exp [-x / \xi]
$$

where

$$
\begin{aligned}
\kappa & =\sqrt{\frac{1}{2} \sqrt{\frac{a_{2}}{c_{2}}}-\frac{c_{1}}{4 c_{2}}} \\
\xi^{-1} & =\sqrt{\frac{1}{2} \sqrt{\frac{a_{2}}{c_{2}}}+\frac{c_{1}}{4 c_{2}}} .
\end{aligned}
$$

In amphiphilic systems $a_{2}$ and $c_{1,2}$ are functions of amphiphile concentration (as well as temperature). In many simple thermodynamical models, short range interactions $k^{2}$ interactions thwarted by others, the modulation length always varies with temperature: in systems with long-range interactions, the modulation length increases as temperature is raised while the converse generically holds for short-range interactions.

When the kernel $v(\vec{k})=k^{4}$, the real space correlator:

$$
G(\vec{x})=-\frac{1}{4 \pi x \sqrt{\mu}} \exp [-x / \xi] \sin \kappa x .
$$

Within the spherical model, this ferromagnetic system displays thermally induced oscillations. At $T=0$ the (ferromagnetic) ground state is unmodulated.

\section{APPENDIX D: THERMODYNAMIC FUNCTIONS IN THE VICINITY OF THE DISORDER LINE}

\section{Non-Abelian Backgrounds}

For a global gauge field normalization, all multipliers $\left\{\mu_{\ell}\right\}_{\ell=1}^{n}=$ const $=\mu$. The internal energy density $U / N=n\left(k_{B} T-\mu\right)$. AS before, the chemical potential $\mu$ may obtained by inverting $1 /\left[k_{B} T\right]=\int \frac{d^{d} k}{(2 \pi)^{d}} G(\vec{k})$. For fixed (non-dynamic) non-Abelian backgrounds, there are no explicit high temperature crossovers- we find no thermodynamic signatures of a disorder line. The number of correlation lengths and modulation lengths is temperature independent. However, the long range physics is sinusoidal (governed by the poles $k_{ \pm}$) at low temperatures while it is nonsinuiodal (dominated by the pole at $k_{0}$ ) at intermediate temperature.

We now examine more complicated gauge theories of glasses. To low orders in perturbation theory, our conclusions are not different. In the $l$-th representation of $S O(3)$ model, the eigenvalues for $k \theta \gg 1$ are

$$
\begin{array}{r}
\lambda_{m}(|\vec{k}|) \approx \\
\theta^{2}\left[(k \theta)^{2}+2 m(k \theta)\right. \\
+\frac{1}{2}\left\{l(l+1)+m^{2}\right\} \\
+\frac{1}{32[2(k \theta)+m+1]}[(l+m)(l-m+1)
\end{array}
$$

$$
\begin{array}{r}
\times(l+m-1)(l-m+2) \\
-(l-m)(l+m+1) \\
\times(l+m+2)(l-m+1)]] .
\end{array}
$$

To this order, the equations

$$
\mu+\lambda_{m}(|\vec{k}|)=0
$$

have a pair of complex conjugate zeros and a single real zero when $\mu>\mu_{\min }$. There is no crossover of a pure imaginary $(k)$ poles into complex ones. The computations are similar for an $S O(4)$ background gauge. When $k \theta \ll 1$, the eigenvalues may be expanded as polynomials in $(k \theta)$. The behavior of the correlation lengths and thermodynamic functions is the same as that observed in the frustrated short range systems that we have discussed- no merger of poles occurs, the number of correlation lengths and the number of modulation lengths are temperature independent. Thus fixed non-Abelian backgrounds do not lead to explicit analytical (continuation) crossover in the thermodynamical functions.

\section{Thermodynamics near the Disorder Line for The Coulomb Frustrated Ferromagnet}

In the spherical $(n \rightarrow \infty)$ limit, the internal energy

$$
\begin{array}{r}
\frac{U}{N}=\frac{1}{2 N} \sum_{\vec{k}}\left[\left\langle|S(\vec{k})|^{2}\right\rangle v(\vec{k})\right], \\
=\frac{1}{2 N} \sum_{\vec{k}} \frac{k_{B} T}{v(\vec{k})+\mu} v(\vec{k})=\frac{1}{2}\left(k_{B} T-\mu\right) .
\end{array}
$$

The chemical potential, $\mu$, may be computed as a function of the temperature $T$ by inverting the spherical equation of state $\frac{1}{k_{B} T}=\frac{1}{n u} \Sigma^{0}(r=\mu)$ with $\Sigma^{0}$ the zeroth order self-energy. $\quad \Sigma^{0}(r=\mu)$ attains different forms for $r^{2}>4 e^{2}$ (when $T>T_{1}$ ) and $r^{2}<4 e^{2}\left(T<T_{1}\right)$. The relation $\tan ^{-1} z=\frac{1}{2 i} \ln \left[\frac{i-z}{i+z}\right]$ may be applied to relate the two natural functional forms. Although there are no divergences at the merger point of the poles $T=T_{1}$, the explicit functional forms of the susceptibility $\left\langle|S(\vec{q})|^{2}\right\rangle=\frac{k_{B} T}{v(\vec{k})+\mu}$ and the specific heat $C_{V}=\frac{\partial U}{\partial T}$ do change. For $T>T_{c}, \mu(T)$ is monotonically increasing in $T$.

$$
\frac{d \mu}{d T}=\frac{1}{T^{2} \Pi(p=0)},
$$

with the zero momentum polarization diagram $\Pi(p=$ $0)=\frac{1}{(2 \pi)^{3}} \int \frac{d^{3} k}{(v(\vec{k})+\mu)^{2}}$. Here we see that no specific heat extrema can be encountered at finite temperatures in any spherical model for $\mu>\mu_{\min }\left(T>T_{c}\right)$. In $\lim n \rightarrow \infty$ (and as shown, also to $O(1 / n)), T_{1}$ is an analytic crossover temperature. As many thermodynamic 
entities are integrals of pair correlators, the change in $G(\vec{x})$ above and below $T_{1}$ suggests a crossover in certain thermodynamic quantities. We expect this crossover to persist to all orders in $1 / n$. We indeed find this to be the case to $O(1 / n)$. The underlying reason for this crossover is very transparent: In the factored propagators $1 /\left[\left(k^{2}+\alpha^{2}\right)\left(k^{2}+\beta^{2}\right)\right]$, the poles have different explicit forms, if $T$ is above or below the disorder line temperature $T_{1}$. The critical temperature $T_{c}(e)$, as computed within the spherical model, drops discontinuously. Above $T_{1}$ we have two correlation lengths. Below $T_{1}$ there exists a single modulation length and a single correlation length. As we have just outlined. even though true long range order does not set it until $T<T_{c}(e)$, an analytical crossover occurs at $T=T_{1}(e)$. At low temperatures this crossover temperature becomes the avoided critical point $T_{c}(0)$. We have suggested that $T_{1}$ can be regarded as an effective dimensional crossover temperature. At lower temperatures the behavior becomes more and more like that of a one dimensional system. At higher temperatures, the behavior is, in most respects, similar to that of a three dimensional nearest neighbor ferromagnet.

\section{APPENDIX E: A GENERALIZED MERMIN-WAGNER INEQUALITY}

\section{The Classical Case}

Our approach is the standard one. We will keep it more general instead of specializing to anti/ferromagnetic order or to interactions of one special sort. With the notations introduced in Section(II), we investigate $n$ component spins on a lattice. An applied magnetic field

$$
\vec{h}(\vec{x})=h \cos (\vec{q} \cdot \vec{x}) \hat{e}_{\alpha}
$$

causes the spins to take on their ground state values.

If $n=\alpha=2$ the unique spiral ground state $\left(\vec{S}^{g}(\vec{x})\right)$, to which a low temperature system would collapse to under the influence of such a perturbation is

$$
S_{1}^{g}(\vec{x})=\sin (\vec{q} \cdot \vec{x}) .
$$

When $n=3$ the ground state is not unique:

$$
\begin{array}{r}
S_{i<n}^{g}(\vec{x})=r_{i} \sin (\vec{q} \cdot \vec{x}) \\
\sum_{i=1}^{n-1} r_{i}^{2}=1
\end{array}
$$

and a magnetic field may be applied along two directions, with all the ensuing steps trivially modified. With the magnetic field applied

$$
H=\frac{1}{2} \sum_{\vec{x}, \vec{y}} \sum_{i=1}^{n} V(\vec{x}-\vec{y}) S_{i}(\vec{x}) S_{i}(\vec{y})-\sum_{\vec{x}} h_{n}(\vec{x}) S_{n}(\vec{x}) .
$$

Note that the knowledge of the ground state is not imperative in providing the forthcoming proof [30]. We exploit the standard rotational invariance of the measure

$$
\int d \mu \cdot=Z^{-1} \int \prod_{\vec{x}} d^{n} S(\vec{x}) \delta\left(S^{2}(\vec{x})-1\right) e^{-\beta H} .
$$

Note that this is not applicable to many other spin models (e.g. Dzyaloshinskii-Moriya interactions). The generators of rotation in the $[\alpha \beta]$ plane are

$$
\begin{gathered}
L_{\alpha \beta} \equiv S_{\alpha} \frac{\partial}{\partial S^{\beta}}-S_{\beta} \frac{\partial}{\partial S^{\alpha}} . \\
0=\frac{d}{d \theta} \int d^{n} S \delta\left(\vec{S}^{2}-1\right) \\
f\left(S_{1}, \ldots, S_{\alpha} \cos \theta+S_{\beta} \sin \theta, \ldots\right. \\
\left., S_{\beta} \cos \theta-S_{\alpha} \sin \alpha, \ldots, S_{n}\right) . \\
0=\int d^{n} S \delta\left(\vec{S}^{2}-1\right) L_{\alpha \beta} f(\vec{S}) .
\end{gathered}
$$

In the up and coming, $\perp$ will denote the the projection along the $\beta$ direction. We define the operators

$$
\begin{array}{r}
\vec{A}(\vec{k}) \equiv \sum_{\vec{x}} \exp [i \vec{k} \cdot \vec{x}] \vec{S}_{\perp}(\vec{x}), \\
\vec{B}(\vec{k}) \equiv \sum_{\vec{x}} \exp [i(\vec{k}+\vec{q}) \cdot \vec{x}] \vec{L}_{\vec{x}}(\beta H) .
\end{array}
$$

By the Schwarz inequality

$$
\left|\left\langle\sum_{i=\alpha, \beta} A_{i}^{*} B_{i}\right\rangle\right|^{2} \leq\left\langle\sum_{i=\alpha, \beta} A_{i}^{*} A_{i}\right\rangle *\left\langle\sum_{i=\alpha, \beta} B_{i}^{*} B_{i}\right\rangle .
$$

We will let $i=\alpha, \beta$ in the sum span a two element subset of the $n$ spin components. For any functional $C$ :

$$
\begin{aligned}
& \vec{L}_{\vec{x}}\left(e^{-\beta H} C\right)=e^{-\beta H}\left\{\vec{L}_{\vec{x}}(C)+C \vec{L}_{\vec{x}}(-\beta H)\right\} . \\
& 0=\int \prod_{\vec{x}} d^{n} S(\vec{x}) \delta\left(\vec{S}^{2}(\vec{x})-1\right) \vec{L}_{\vec{x}}\left[e^{-\beta H} C\right]
\end{aligned}
$$

$$
\langle C B(\vec{p})\rangle=\left\langle\sum_{\vec{x}} \exp [i \vec{p} \cdot \vec{x}] \vec{L}_{\vec{x}}(C)\right\rangle
$$

$$
\begin{array}{r}
\sum_{i=\alpha, \beta}\left\langle L_{\vec{x}}^{i}\left(L_{\vec{y}}^{i}(\beta H)\right)\right\rangle=\beta\left\langle\left[\sum_{i=\alpha, \beta} S_{i}(\vec{x}) S_{i}(\vec{y}) V(\vec{x}-\vec{y})\right.\right. \\
\left.\left.-h(\vec{x}) S_{n}(\vec{x})\right]\right\rangle
\end{array}
$$

$$
\begin{array}{r}
\left\langle\vec{B}(\vec{k})^{*} \cdot \vec{B}(\vec{k})\right\rangle=\beta \sum_{\vec{x}, \vec{y}}\{(\cos (\vec{k}+\vec{q}) \cdot(\vec{x}-\vec{y})-1) \\
\left.\left[\sum_{i=\alpha, \beta}\left\langle S_{i}(\vec{x}) S_{i}(\vec{y})\right\rangle\right] V(\vec{x}-\vec{y})\right\} \\
-h(\vec{x})\left\langle S_{n}(\vec{x})\right\rangle \geq 0
\end{array}
$$


Henceforth, for simplicity, we specialize to $n=2$. Fourier expanding the interaction kernel

$$
V(\vec{x}-\vec{y})=\frac{1}{N} \sum_{\vec{t}} v(\vec{t}) e^{i \vec{t} \cdot(\vec{x}-\vec{y})},
$$

and substituting

$$
\langle\vec{S}(\vec{x}) \cdot \vec{S}(\vec{y})\rangle=\frac{1}{N^{2}} \sum_{\vec{u}}\left\langle|\vec{S}(\vec{u})|^{2}\right\rangle e^{i \vec{u} \cdot(\vec{x}-\vec{y})},
$$

we obtain

$$
\begin{array}{r}
0 \leq\left\langle\vec{B}(\vec{k})^{*} \cdot \vec{B}(\vec{k})\right\rangle \equiv \beta \Delta_{\vec{k}}^{(2)} E-\beta h_{\vec{q}}\left\langle S_{n}(-\vec{q})\right\rangle= \\
\frac{\beta}{2 N} \sum_{\vec{u}}[v(\vec{u}+\vec{k})+v(\vec{u}-\vec{k}) \\
-2 v(\vec{u})]\left\langle\left.\vec{S}(\vec{u})\right|^{2}\right\rangle-\beta h_{\vec{q}}\left\langle S_{n}(-\vec{q})\right\rangle
\end{array}
$$

where $\Delta_{\vec{k}}^{(2)} E$ measures the finite difference of the internal energy with respect to a boost of momentum $\vec{k}$.

$$
\begin{gathered}
\left\langle\vec{A}(\vec{k})^{*} \cdot \vec{A}(\vec{k})\right\rangle=\sum_{\vec{x}, \vec{y}}\left\langle\vec{S}_{\perp}(\vec{x}) \cdot \vec{S}_{\perp}(\vec{y})\right\rangle \\
\times \exp [i \vec{k} \cdot(\vec{x}-\vec{y})] . \quad(\mathrm{E} 19) \\
\left\langle\vec{A}(\vec{k})^{*} \cdot \vec{B}(\vec{k})\right\rangle=\left\langle\sum_{i, \vec{x}} L_{\vec{x}}^{i}\left(\vec{S}_{\perp}(\vec{x})\right) \exp [i(\vec{k}+\vec{q}) \cdot \vec{x}]\right\rangle=m_{\vec{q}},
\end{gathered}
$$

where $m_{\vec{q}} \equiv\left\langle S_{n}(\vec{q})\right\rangle$ and, as noted earlier, $\perp$ refers to the $i=1$ spin direction orthogonal to $i=n=2$. Note that with our convention for the Fourier transformations, a macroscopically modulated state of wave-vector $\vec{q}$, the magnetization $m_{q}=\mathcal{O}(N)$ as is the energy difference in Eqn.(E18). Trivially rewriting the Schwarz inequality and summing over all momenta $\vec{k}$,

$$
\sum_{\vec{k}} \frac{\left\langle\vec{A}(\vec{k})^{*} \cdot \vec{B}(\vec{k})\right\rangle}{\left\langle\vec{B}(\vec{k})^{*} \cdot \vec{B}(\vec{k})\right\rangle} \leq \sum_{\vec{k}}\left\langle\vec{A}(\vec{k})^{*} \cdot \vec{A}(\vec{k})\right.
$$

which explicitly reads

$$
\begin{array}{r}
2 N\left|m_{\vec{q}}\right|^{2}\left(\beta \sum _ { \vec { k } } \left(\left\langle|\vec{S}(\vec{u})|^{2}\right\rangle[v(\vec{k}+\vec{u})\right.\right. \\
\left.+v(\vec{u}-\vec{k})-2 v(\vec{u})]+2|h|\left|m_{q}\right|\right)^{-1} \\
\leq N \sum_{\vec{k}} \sum_{\vec{x}, \vec{y}}\left\langle\vec{S}_{\perp}(\vec{x}) \cdot \vec{S}_{\perp}(\vec{y})\right\rangle e^{i \vec{k} \cdot(\vec{x}-\vec{y})} \\
=N \sum_{\vec{x}}\left\langle\vec{S}_{\perp}^{2}(\vec{x})\right\rangle .
\end{array}
$$

Explicitly, as the integral $\int^{|\vec{k}|>\delta} \frac{d^{d} k}{(2 \pi)^{d}} \ldots$ is non-negative (as $\left\langle\vec{B}(\vec{k})^{*} \cdot \vec{B}(\vec{k})\right\rangle \geq 0$ the denominator in Eqn.(E21) is positive for each individual value of $\vec{k})$, and as $\left\langle\vec{S}_{\perp}^{2}(\vec{x})\right\rangle \leq$ 1 , we obtain in the thermodynamic limit

$$
\begin{array}{r}
\frac{2}{\beta}\left|m_{\vec{q}}\right|^{2} \int^{|\vec{k}|<\delta} \frac{d^{d} k}{(2 \pi)^{d}}\left[\int \frac{d^{d} u}{(2 \pi)^{d}}\right. \\
\left\langle|\vec{S}(\vec{u})|^{2}\right\rangle(v(\vec{k}+\vec{u})+v(\vec{k}-\vec{u})-2 v(\vec{u})) \\
\left.+2|h|\left|m_{q}\right|\right]^{-1} \leq 1 .
\end{array}
$$

Taking $\delta$ to be small we may bound from above (for each value of $\vec{k}$ ) the positive denominator in the square brackets and consequently

$$
\begin{array}{r}
\int^{|\vec{k}|<\delta} \frac{d^{d} k}{(2 \pi)^{d}}\left[\int \frac{d^{d} u}{(2 \pi)^{d}}\right. \\
\left\langle|\vec{S}(\vec{u})|^{2}\right\rangle(v(\vec{k}+\vec{u})+v(\vec{k}-\vec{u})-2 v(\vec{u})) \\
\left.+2|h|\left|m_{q}\right|\right]^{-1} \geq \int^{|\vec{k}|<\delta} \frac{d^{d} k}{(2 \pi)^{d}}\left[\int \frac{d^{d} u}{(2 \pi)^{d}}\right. \\
\left.A_{1} k^{2} \lambda_{\vec{u}}\left\langle|\vec{S}(\vec{u})|^{2}\right\rangle+2|h|\left|m_{q}\right|\right]^{-1},
\end{array}
$$

with $\lambda_{\vec{u}}$ chosen to be the largest principal eigenvalue of the $d \times d$ matrix $\partial_{i} \partial_{j}[v(\vec{u})]$, and $A_{1}$ a constant. For a twice differentiable $v(\vec{u})$, and for $|\vec{k}| \leq \delta$ where $\delta$ is finite,

$$
(v(\vec{k}+\vec{u})+v(\vec{k}-\vec{u})-2 v(\vec{u})) \leq A_{1} \lambda_{\vec{u}} k^{2} \leq B_{1} k^{2}
$$

for all $\vec{u}$ within the Brillouin Zone with the additional positive constant $B_{1}$ introduced [31]. Here we reiterate that $\left\langle\vec{B}^{*}(\vec{k}) \cdot \vec{B}(\vec{k})\right\rangle$ of Eqn.(E18) is positive definite and consequently the bound derived is powerful.

In $d \leq 2$, the integral $\int^{|\vec{k}|<\delta} \frac{d^{d} k}{B_{1} k^{2}}$ diverges making it possible to satisfy eqn.(E22), at finite temperatures, when the external magnetic field $h \rightarrow 0$ only if the magnetization $m_{q}=0$. If finite size effects are restored, in a system of size $N=L \times L$ where the infrared cutoff in the integral is $\mathcal{O}\left(\frac{2 \pi}{L}, \frac{2 \pi}{L}\right)$ the latter integral diverges as $\mathcal{O}(\ln N)$. This implies that the upper bound on $\left|m_{\vec{q}}\right|$ scales as $\mathcal{O}(N / \sqrt{\ln N})$, much lower than the $\mathcal{O}(N)$ requisite for finite on-site magnetization. For further details see [30]. If there are $M \geq 2$ pairs of minimizing modes and $2 p+1 \geq n \geq 2 p$ (with an integer $p$ ) then we may apply an infinitesimal symmetry breaking magnetic field along, at most, $\min \{p, M\}$ independent spin directions $(\alpha)$. Employing the spin rotational invariance within each plane $[\alpha \beta]$ associated with any individual mode $\vec{\ell}$ (au lieu of a specific $\vec{q}$ ) we may produce a bound similar that in Eqn.(E22) wherein $\left\langle|\vec{S}(\vec{u})|^{2}\right\rangle$ will be replaced by $\sum_{i=\alpha, \beta}\left\langle\left|S_{i}(\vec{u})\right|^{2}\right\rangle$ and $\left|m_{\vec{q}}\right| \rightarrow\left|m_{\alpha}(\vec{\ell})\right|$.

\section{The Quantum Case}

The finite temperature behavior of a quantum system is, in many respects, similar to that of a classical system. 
The quantum system is also invariant under rotations with $\hat{S}^{2}(\vec{x})=S(S+1)$.

Alternatively, one could directly tackle the $n=3$ quantum case by applying the Bogoliubov inequality

$$
\frac{\beta}{2}\left\langle\left\{A, A^{\dagger}\right\}\right\rangle *\left\langle\left[[C, H], C^{\dagger}\right]\right\rangle \geq|\langle[C, A]\rangle|^{2}
$$

with $[$,$] and \{$,$\} the commutator and anticommu-$ tator respectively. From this inequality, it follows that $\left\langle\left[[C, H], C^{\dagger}\right]\right\rangle$ is positive definite. In particular, for any six operators $\left\{A_{1}, A_{2}, A_{3}, C_{1}, C_{2}, C_{3}\right\}$,

$$
\begin{array}{r}
\frac{\beta}{2}\left(\sum_{a=1}^{3}\left\langle\left\{A_{a}, A_{a}^{\dagger}\right\}\right\rangle\right)\left(\sum_{a}\left\langle\left[\left[C_{a}, H\right], C_{a}^{\dagger}\right]\right\rangle\right) \geq \\
\sum_{a}\left|\left\langle\left[C_{i}, A_{i}\right]\right\rangle\right|^{2}
\end{array}
$$

Setting $A_{1}=S_{2}(\vec{q}-\vec{k})$ and $C_{1}=S_{1}(\vec{k})$ we will once again obtain Eqn(E22) with the classical spins replaced by their quantum counterparts.

Rather explicitly, employing

$$
\left[S^{\alpha}(\vec{k}), S^{\beta}\left(\vec{k}^{\prime}\right)\right]=i \epsilon^{\alpha \beta \gamma} S_{\gamma}\left(\vec{k}+\vec{k}^{\prime}\right),
$$

we find for the Hamiltonian of Eqn.(E4) (with $n=3$ ),

$$
\begin{array}{r}
{\left[\left[C_{1}, H\right], C_{1}^{\dagger}\right]=\frac{1}{2 N} \sum_{\vec{k}^{\prime}}\left(S_{2}\left(\vec{k}^{\prime}\right) S_{2}\left(-\vec{k}^{\prime}\right)+S_{3}\left(\vec{k}^{\prime}\right) S_{3}\left(-\vec{k}^{\prime}\right)\right)} \\
\times\left[v\left(\vec{k}+\vec{k}^{\prime}\right)-2 v(\vec{k})+v\left(\vec{k}^{\prime}-\vec{k}\right)\right] \\
+\frac{1}{N} \sum_{\vec{k}^{\prime}} h_{3}\left(\vec{k}^{\prime}\right) S_{3}\left(-\vec{k}^{\prime}\right) .
\end{array}
$$

Similarly,

$$
\left|\left\langle\left[C_{1}, A_{1}\right]\right\rangle\right|^{2}=\left|\left\langle S_{3}(\vec{q})\right\rangle\right|^{2},
$$

the squared magnetization along the $\mathrm{z}$ (or 3) direction for a mode $\vec{q}$, and

$$
\begin{array}{r}
\sum_{\vec{k}}\left\langle\left\{A_{1}, A_{1}^{\dagger}\right\}\right\rangle=2 \sum_{\vec{k}}\left\langle S_{2}(\vec{k}) S_{2}(-\vec{k})\right\rangle \\
=2\left\langle\left[S_{2}(\vec{x}=0)\right]^{2}\right\rangle .
\end{array}
$$

Next, let us cyclically set, $A_{2}=S_{3}(\vec{q}-\vec{k}), C_{2}=$ $S_{2}(\vec{k}), A_{3}=S_{1}(\vec{q}-\vec{k})$, and $C_{3}=S_{3}(\vec{k})$. The commutators associated with these operators are all identically the same apart from a uniform cyclic permutation of all spin components involved.

Trivially rewriting the symmetrized Bogoliubov inequality Eqn.(E26) and summing over all modes $\vec{k}$,

$$
\frac{\beta}{2} \sum_{\vec{k}, a}\left\langle\left\{A_{a}, A_{a}^{\dagger}\right\}\right\rangle \geq \sum_{\vec{k}} \frac{\sum_{a}\left|\left\langle\left[C_{a}, A_{a}\right]\right\rangle\right|^{2}}{\sum_{a}\left\langle\left[\left[C_{a}, H\right], C_{a}^{\dagger}\right]\right\rangle} .
$$

Replacing the $\vec{k}$ sums by integrals in the thermodynamic limit, and employing the positivity of $\left\langle\left[\left[C_{a}, H\right], C_{a}^{\dagger}\right]\right\rangle$ that follows from the Bogoliubov inequality for each individual value of $\vec{k}$, we find the trivial quantum analogue of Eqn.(E22),

$$
\begin{array}{r}
\frac{1}{2 \beta}\left|m_{\vec{q}}\right|^{2} \int^{|\vec{k}|<\delta} \frac{d^{d} k}{(2 \pi)^{d}}\left[\int \frac{d^{d} u}{(2 \pi)^{d}}\right. \\
\left\langle|\vec{S}(\vec{u})|^{2}\right\rangle(v(\vec{k}+\vec{u})+v(\vec{k}-\vec{u})-2 v(\vec{u})) \\
\left.+|h|\left|m_{q}\right|\right]^{-1} \leq S(S+1) .
\end{array}
$$

Apart from the simple scaling factor of $4 S(S+1)=$ $2 \sum_{a}\left\langle\left\{A_{a}, A_{a}^{\dagger}\right\}\right\rangle$ by comparison to the classical case, there is no difference between this inequality and its classical counterpart in the zero field limit. We symmetrized the Bogoliubov inequality (Eqn.(E26)) in order to avoid the appearance of only two transverse spin components in the Fourier weights $\left\langle\left|S_{i}(\vec{q})\right|^{2}\right\rangle$ so as to give the resulting Mermin-Wagner inequality a transparent physical meaning associated with the energy boost differences $\Delta_{\vec{k}}^{(2)} E$ which are symmetric in all spin indices. From here onward the discussion can proceed as in the classical case.

\section{APPENDIX F: MERMIN-WAGNER BOUNDS IN HIGH DIMENSIONS}

In any dimension, Eqn.(E22) reads in the limit $h \rightarrow 0$

$$
2\left|m_{\vec{q}}\right|^{2} T \int \frac{d^{d} k}{(2 \pi)^{d}} \frac{1}{\Delta_{\vec{k}}^{(2)} E} \leq 1
$$

with the shorthand defined by Eqn.(E18). By parity invariance and noting that $\{\vec{S}(\vec{x})\}$ are real,

$$
\sum_{\vec{u}} v(\vec{k}+\vec{u})\left\langle|\vec{S}(\vec{u})|^{2}\right\rangle=\sum_{\vec{u}} v(\vec{k}-\vec{u})\left\langle|\vec{S}(\vec{u})|^{2}\right\rangle .
$$

Thus the denominator of Eqn.(F1) reads $\Delta_{\vec{k}}^{(2)} E=$ $\left[2\left(E_{\vec{k}}-E_{0}\right)\right]$ where $E_{\vec{k}}$ is the internal energy of system after undergoing a boost of momentum $\vec{k}$ and $E_{0}$ denotes the internal energy of the un-boosted system. In some instances when the dispersion relation about an assumed zero temperature ground state is inserted into Eqn.(F1), we will find that the integral in Eqn.(F1) diverges: At arbitrarily low temperatures we cannot assume the zero temperature ground state with the natural dispersion relation $\Delta E$ for fluctuations about it. A case in point is the dispersion relation for the Coulomb Frustrated Ferromagnet. The denominator in Eqn.(E22) is a finite temperature extension of the $T=0$ dispersion relations $\left(\Delta_{\vec{k}}^{(2)} E\right)$. In general, at zero temperature,

$$
\left\langle|\vec{S}(\vec{k})|^{2}\right\rangle=\frac{N^{2}}{2}\left[\delta_{\vec{k}, \vec{q}}+\delta_{\vec{k},-\vec{q}}\right]
$$


and the integral in Eqn.(F1) becomes Eqn.(35).

Whenever Eqn.(35) diverges, an assumption of an almost ordered ground state at arbitrarily low temperatures $\left(T=0^{+}\right)$with the ensuing zero temperature dispersion relation $\left(\Delta_{\vec{k}}^{(2)} E=\left[2\left(E_{\vec{k}}-E_{0}\right)\right]\right)$ about that ordered state is flawed: Eqn.(F1) is strongly violated. This poses no problem for most canonical $d>2$ dimensional models where the integral in Eqn.(35) is finite. For the three dimensional Coulomb Frustrated Ferromagnet and other high dimensional models the divergence of this integral hints possible non-trivialities. The average decoherence (or relaxation) time scale $\langle\tau\rangle$ may diverge if we assume a zero temperature dispersion of fluctuations about an ideal crystal. A divergent decoherence time scale suggests the absence of broken translational symmetry. Formally,

$$
\int \frac{d^{3} k}{(2 \pi)^{3}} \tau_{k}=\tau(\vec{r}=0)
$$

In order for magnetization $m_{q}=\mathcal{O}(N)$ to arise, the average of the inverse boost energy over all of $\vec{k}$ space

$$
\tau \equiv\left\langle\frac{1}{\Delta_{\vec{k}}^{(2)} E}\right\rangle_{\vec{k}} \leq \mathcal{O}\left(T^{-1}\right),
$$

at all $0<T<T_{c}$. In most $d>2$ systems this is trivially satisfied with the average bounded by a constant at zero temperature. This average diverges at $T=0$ whenever Eqn.(35) does. There are two possibilities:

(i) The system is ordered at all temperatures $T<T_{c}$ in which case, the thermodynamic average of Eqn.(F5) is finite for all $T>0$ and $\tau$ is non-analytic at $T=0$.

(ii) The system is disordered at all finite temperatures and orders classically only at $T=0$.

The first possibility ((i)) was argued for by a nonrigorous yet elegant diagrammatic analysis by Brazovskii [35] long ago: Thermal fluctuations, on their own, may enhance (or generate) cubic terms fortifying (or triggering weak) first order transitions. This cannot be ruled out by the rigorous Mermin-Wagner inequalities that we derived. As reiternated, if the fluctuation integral diverges then we may not obtain the low temperature $T=0^{+}$ dispersion by assuming a nearly perfectly ordered state. This does not rigorously preclude order. Order, if it exists at arbitrarily low temperatures must display non-trivial excitation spectra about it. If such a possibility arises, it might be immaterial if the system is permanently frozen into a glass before reaching an equilibrium thermodynamic transition. In both cases, $\tau(T)$ is non-analytic at $T=0$. The integral of Eqs. $(35, \mathrm{~F} 5)$ has a suggestive physical interpretation. If the quantum spin system is subjected to a boost of momentum $\vec{k}$, then

$$
1 / \Delta_{\vec{k}}^{(2)} E \equiv \tau_{\vec{k}}
$$

is the characteristic lifetime of the excited state. The average in Eqn.(F5) is the characteristic relaxation (or decoherence) time of the system averaged over magnons of all possible momenta. Whenever the average characteristic relaxation time

$$
\begin{array}{r}
\left\langle\tau_{\vec{k}}\right\rangle=\int \frac{d^{d} k}{(2 \pi)^{d}}\left[\int \frac{d^{d} u}{(2 \pi)^{d}}\right. \\
\left.\left\langle|\vec{S}(\vec{u})|^{2}\right\rangle(v(\vec{k}+\vec{u})+v(\vec{k}-\vec{u})-2 v(\vec{u}))\right]^{-1}
\end{array}
$$

diverges then by our generalized inequality

$$
2\left|m_{q}\right|^{2} T\left\langle\tau_{\vec{k}}\right\rangle=2\left|m_{q}\right|^{2} T \tau_{r=0} \leq 1
$$

the system does not order in such a way that the fluctuation dispersion about any viable ground state is valid. Moreover, as we show in the text the system displays divergent glassy dynamics. The characteristic divergent relaxation times in glassy systems suggest a divergent decoherence time and an inability to write a wave function of a quantum glass. The glassy state may be specified by a density matrix [36].

* Present address: Theoretical Division, Los Alamos National Laboratory, Los Alamos, NM 87545

[1] J. F. Sadoc and R. Mosseri, "Geometrical Frustration", Cambridge University Press (1999), and references therein

[2] F. C. Frank and J. S. Kasper, Acta Crystallogr. 11, 184 (1958), 12, 483 (1959)

[3] D. R. Nelson, Phys. Rev. B 28, 5515 (1983)

[4] J. F. Sadoc, J. de Physique Lett., 44, L-707 (1983)

[5] J. P. Sethna, Phys. Rev. B 31, 6278 (1985)

[6] J. F. Sadoc and J. Charvolin, J. de Physique, 47, 683 (1986)

[7] S. Sachdev and D. R. Nelson, Phys. Rev. B, 32, 1480 (1985)

[8] In metallic glasses, the interface energy between the solid and liquid allows supercooling in the absence of extraneous components. X-ray diffraction on levitated supercooled drops [9] confirms icosahedral order.

[9] K. F. Kelton et al. Phys. Rev. Lett. 90, 195504 (2003); T. Schenk et al. 89, 75507 (2002)

[10] L. Chayes, V. J. Emery, S. A. Kivelson, Z. Nussinov, and G. Tarjus, Physica A 225, 129 (1996)

[11] Z. Nussinov, J. Rudnick, S. A. Kivelson, and L. N. Chayes, Phys. Rev. Lett. 83, 472 (1999)

[12] D. Kivelson, S. A. Kivelson, X. L. Zhao, Z. Nussinov, and G. Tarjus, Physica A 219, 27 (1995)

[13] D. R. Nelson and M. Widom, Nucl. Phys. B 240, 113 (1984)

[14] In a similar spirit, if we demand the matching, upon rolling, of ideal wave-functions present on spheres, the action would be the same with $\rho$ replaced by a "wavefunction" 


$$
\Psi(\vec{x}, \hat{n}) \equiv \sum_{\ell, m} Q_{\ell, m}(\vec{x}) Y_{\ell}^{m}(\hat{n})
$$

The global normalization reads:

$$
1=\int d^{2} x \sum_{\ell, m}\left|Q_{\ell, m}(\vec{x})\right|^{2},
$$

which is, en effect, the spherical constraint of the large $n$ spin models which we will come back to later on. The Hamiltonian will be the same with the kinetic energy term $\left(-\nabla^{2} \Psi\right)$ replaced a squared covariant derivative $\left(-D^{2} \Psi\right)$. All stated can be applied to such problems.

[15] N. Rivier, Phil. Mag. B, 40, 859 (1979); N. Rivier, The Taniguchi Symphosium on The Nature of Topological Disorder, F. Yonezawa and T. Ninomiya, editors, Springer Verlang, 14 (1983)

[16] D. Nelson and F. Spaepen, Solid State Phys. 42, 1 (1989)

[17] R. Mosseri and J. F. Sadoc, J. Phys. Lett. (Paris), 45, L827 (1984)

[18] J. F. Sadoc and F. Mosseri, "Amorphous Materials" edited by V. Vitek (American Institute of Metallurgical Engineering, New York, 1983), p. 111

[19] W. Klein, H. Gould, R. A. Ramos, I. Clejan, and A. I. Mel'uck, Physica A 205, 738 (1994)

[20] J. O. K. Doye, D. J. Wales, F. H. Zetterling, and M. Dzugutov, cond-mat/0205374

[21] J. Schmalian and P. G. Wolynes, Phys. Rev. Lett. 85, 836 (2000); H. Westfahl, Jr., J. Schmalian, and P. G. Wolynes, Phys. Rev. B 64, 174203 (2001)

[22] K. K. Loh et al., cond-mat/0206494

[23] S. Wu, J. Schmalian, G. Kotliar, P. Wolynes, condmat/0305404

[24] Z. Nussinov, thesis, University of California, Los Angeles (1999)

[25] T. R. Kirkpatrick and P. G. Wolynes, Phys. Rev. B 35, 3072 (1987); 36, 8552 (1987); T. R. Kirkpatrick and P. G. Wolynes, Phys. Rev. A 40, 1045 (1989); T. R. Kirkpatrick and D. Thirumalai, Phys. Rev. Lett. 58, 2091 (1987)

[26] F. C. Frank, Proc. Royal Soc., London, A 215, (1952) 43

[27] M. Grousson, G. Tarjus, and P. Viot, cond-mat/0111305

[28] P. L. Geissler and D. R. Reichman, cond-mat/0304254

[29] J. Schmalian, S. Wu, P. G. Wolynes, cond-mat/0305420

[30] Note that even if we had not known the ground states we could still prove that there is no magnetization. All we need to do is to apply an infinitesimal magnetic field

$$
\vec{h}(\vec{x})=h \vec{S}^{\text {ground-state }}(\vec{x})
$$

with as yet an unknown ground state $\vec{S}^{\text {ground-state }}(\vec{x})$. Replacing any appearance of the minimizing wave-vector $\vec{q}$ in the Schwarz inequality and the definition of $B(\vec{k})$ by the more general wave-vector $\vec{\ell}$ and setting $h \rightarrow 0^{+}$one would arrive at the conclusion that $m_{\vec{\ell}}=0$ for each mode $\vec{\ell}$. Consequently,

$$
\left\langle S_{n}(\vec{x})\right\rangle=\frac{1}{N} \sum_{\vec{\ell}} e^{i \vec{l} \cdot \vec{x}} m_{\ell}=0
$$

Stated alternatively, by Parseval's theorem,

$$
\sum_{\vec{x}}\langle\vec{S}(\vec{x})\rangle^{2}=\frac{1}{N^{2}} \sum_{\vec{l}}\langle\vec{S}(\vec{l})\rangle\langle\vec{S}(-\vec{l})\rangle .
$$

Taking careful note of the system size $(N)$ in the fluctuation integrals and the $N$ terms in the summand of Eqn.(F13),

$$
\sum_{\vec{x}}\langle\vec{S}(\vec{x})\rangle^{2} \leq \mathcal{O}\left(\frac{N}{\ln N}\right)
$$

and $|\langle\vec{S}(\vec{x})\rangle|$ at any site $\vec{x}$ diminishes as $\mathcal{O}(\sqrt{1 / \ln N})$, vanishing in the thermodynamic $(N \rightarrow \infty)$ limit.

[31] More precisely, everything to be claimed holds for the more general class of functions $v(\vec{k})$ for which $\max _{\vec{u} \in B . Z .}[v(\vec{k}+\vec{u})+v(\vec{k}-\vec{u})-2 v(\vec{u})] \leq B_{1} k^{2}$ is obeyed for all momenta $\vec{k}$ with some finite positive constant $B_{1}$.

[32] H. Kleinert, "Gauge Fields in Condensed Matter", World Scientific (1989)

[33] Z. Nussinov, cond-mat/0105253

[34] S. Alexander and J. P. McTague, Phys. Rev. Lett. 41, 702 (1978)

[35] S. Brazovskii, Sov. Phys. JETP 41, 85(1975)

[36] I am indebted to Joerg Schmallian for this observation 